\title{
Gene editing in Brassica napus for basic research and trait development
}

\author{
Greg F. W. Gocal ${ }^{1}$ (1)
}

Received: 7 June 2021 / Accepted: 17 June 2021 / Published online: 9 August 2021 / Editor: David Songstad

(C) The Author(s) 2021

\begin{abstract}
The genome of Brassica napus L. is the result of several polyploidization events that occurred during the history of B. napus. Due to its relatively short domestication history, diversity is relatively limited. An increasing number of loci in this crop's genome have been gene-edited using various technologies and reagent delivery methods for basic research as well as for trait development. New alleles have been developed as edits in single, 2, 4, or more homologous loci in this important oilseed crop. This comprehensive review will summarize new alleles that have been developed as they relate to weed control, flowering, selfincompatibility, plant hormone biology, disease resistance, grain composition, and pod shatter reduction. These new alleles have significantly augmented our understanding of both plant growth and development for basic research as well as for their potential commercial impacts.
\end{abstract}

Keywords Brassica napus $\cdot$ Gene editing $\cdot$ Pod shatter $\cdot$ Herbicide tolerance $\cdot$ Flowering $\cdot$ Hormone signaling $\cdot$ Disease resistance

\section{Introduction}

Genomic variation CRISPR/Cas9 has already proven to be a powerful tool for affecting numerous plant traits that have the potential to add diversity to existing organisms while offering significant benefits to plant breeding programs focused on different areas of crop improvement. One such example comes from the work of Rodríguez-Leal et al. (2017) who used a gene editing approach to develop a rich source of allelic diversity for breeding in tomato. In this way, gene editing technologies are a logical extension of plant breeding and can be applicable across a wide range of plants (van Eck 2020).

Genome variation is the primary source of phenotypic trait expression in plants. Farmers and plant breeders have harnessed existing genetic diversity using many different methods over thousands of years, beginning with mass selection to identify and select better performing plants, utilizing a better understanding of genetics and inheritance to develop modern plant breeding approaches, and more recently the application of advanced biotechnology tools such as genetic

Greg F. W. Gocal

ggocal@cibus.com

1 Cibus US LLC, 6455 Nancy Ridge Drive, San Diego, CA 92121, USA markers, tissue culture, and molecular biology, among other technological advancements. Plants have evolved complex genomes to be able to better adapt to challenges in the environment and evolve as a species. In some cases, the evolution of gene families is characterized by gene duplication resulting from polyploidization, tandem gene duplication, and segmental duplication. The outcomes of all these events are potentially better adapted organisms for specific growing environments.

The source of variation utilized by plant breeders for trait development is in the existing diversity of the target species and its ancestors. Conventional plant breeding methods involve the evaluation and selection of potential parental plant characteristics, with the goal of combining those traits leading to improved performance. Additionally, the breeder will be interested in selecting for traits which may add special characteristics like improved quality or herbicide tolerance to add to the overall yield performance. The process of plant breeding can take several generations of crossing, selfing, and selection to achieve the desired outcome, which can take many years or decades to achieve. The specific length of time depends on the biology of the crop (e.g., length of a typical growth cycle) and the complexity of the trait combinations that are sought. The more complex the trait combination, the longer and more difficult the process. Identifying and selecting an elite combination of genetics in a single variety 
can take up to $10 \mathrm{yr}$ for row crop species. This presents a significant challenge as our population grows, increasing the need for sustainably produced food. In some cases, the desired trait does not exist in the elite breeding population or is present but very difficult to select because the trait requires several different genes interacting appropriately in order to be expressed. If multiple genes exist to define the desired trait, plant breeders will use several cycles of backcrossing to move the trait into elite genetic backgrounds, although the more complex traits may also lead to increased linkage drag from the donor material. If the desired trait does not exist in the elite material forming the core of the breeding program, laboratory tools such as inter-species crossing, mutagenesis, transgenic development, or gene editing can be used to assist in developing the trait expression of interest or to create new genetic combinations. As will be detailed in this review article focused on Brassica napus, several gene editing tools have been used including CRISPR/Cas9 to develop loss of function (LOF) alleles for various loci and base editing to enhance diversity towards developing traits in this important oilseed crop.

Brassica napus: a crop for trait development using gene editing The genus Brassica consists of extensively agronomically diverse species. The triangle of $U$ (Figure 1) summarizes the interspecific hybridization events between diploid progenitors B. rapa (syn. campestris; AA), B. nigra (BB), and $B$. oleracea $(\mathrm{CC})$ resulting in the allotetraploids, B. juncea (AABB), B. carinata (BBCC), and B. napus (AACC) (Nagaharu et al. 1935; Snowdon et al. 2002). The natural crossing of $B$. rapa (AA; $\mathrm{n}=18$ ) by $B$. oleracea $(\mathrm{CC}$; $\mathrm{n}=20$ ) that led to $B$. napus (AACC; $\mathrm{n}=38$ ) occurred approximately 7,500 yr ago (Chalhoub et al. 2014). B. napus is the result of a process of 72 genome multiplication events $(\times 3[\gamma]$ $\times 2[\alpha] \times 2[\beta] \times 3 \times 2$ ) since the origin of angiosperms. Most recently, this includes the whole genome triplication event characteristic of the Brassicaceae tribe and a duplication in the coming together of $B$. rapa and B. oleracea (Chalhoub et al. 2014). Oilseed canola include B. rapa, B. napus, and $B$. juncea, varieties with international standards for erucic acid and glucosinolate contents (Sharafi et al. 2015). B. napuscanola, as it is known in North America, or oilseed rape (OSR), as it is known in Europe - is cultivated mainly for its oil-rich seed, the third largest source of vegetable oil in the world (https://apps.fas.usda.gov/psdonline/circulars/oilseeds. pdf). Its oil can also be used for industrial purposes and the meal, as a byproduct of the crush, is used as animal feed - principally in poultry and dairy operations. Canola is the second most profitable oilseed row crop, ranking only behind soybean in production and value (Foreign Agricultural Service/USDA, 2021). There are two types of OSR, spring (SOSR) and winter (WOSR). SOSR, as its name indicates, is planted in the spring and harvested in the fall. By comparison, WOSR is planted in the late summer or fall,
Figure 1 The triangle of $\mathrm{U}$ describes the origin of the allotetraploid species $B$. juncea (AABB), B. carinata (BBCC), and B. napus (AACC) as a result of interspecific hybridization events between the diploid progenitors $B$. rapa (AA), B. nigra (BB), and B. oleracea (CC).

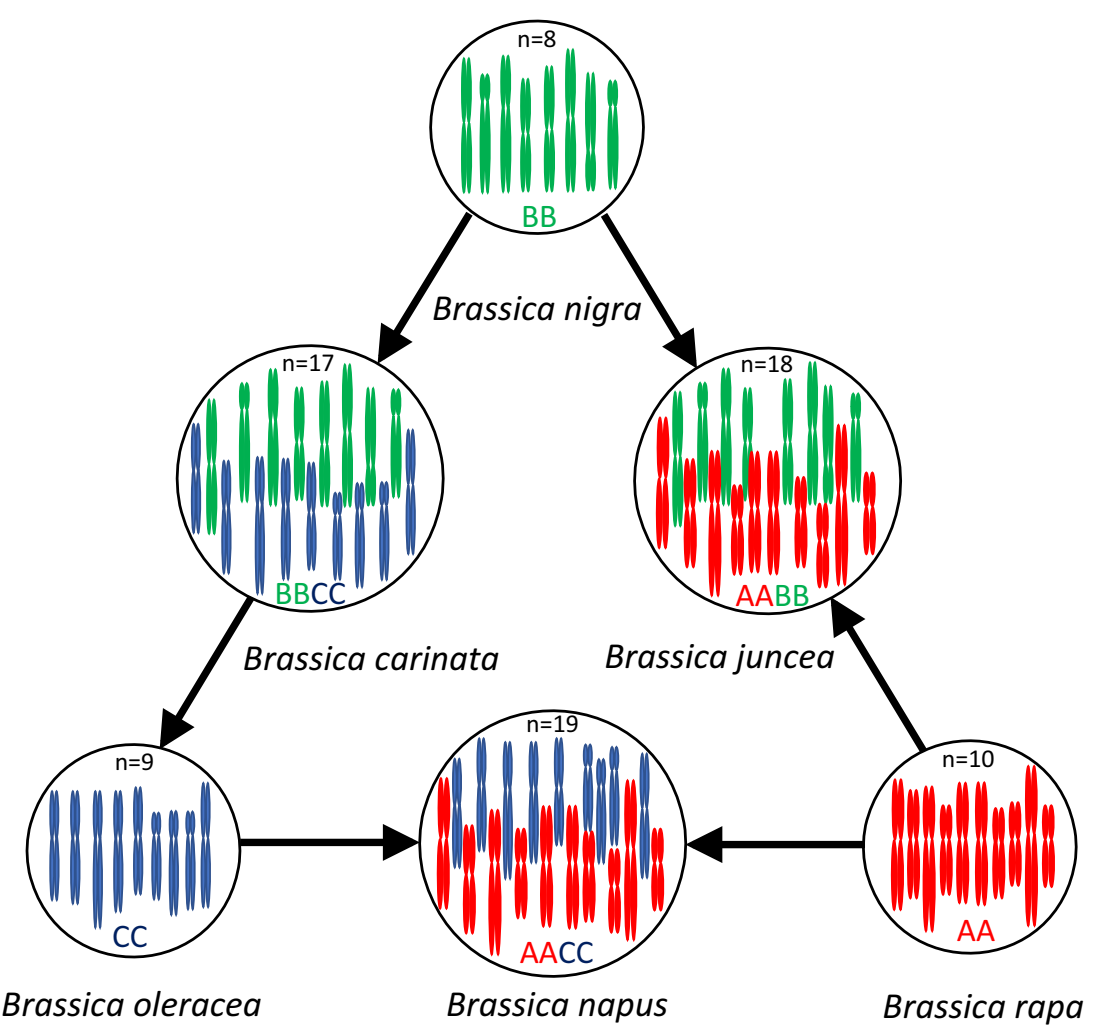


germinates with the seedling establishing before winter sets in, and then in the spring continues its growth culminating with flowering, seed set, and harvest in the following summer. SOSR types are grown in the Americas, Australia, and northern Europe, whereas WOSR is primarily grown in Europe and China. This review will focus on gene editing of OSR.

Trait development opportunities While gene editing technologies have seemingly unlimited potential to develop novel traits, one key element of deploying these tools is to have a deep understanding of potentially complex trait architectures. Knowing what to edit is a challenge for trait developers to be able to adapt gene editing into a tool for breeders to deploy on a routine basis. Due to significant recent advances in sequencing technologies, computational biology, and bioinformatics, the fields of functional genomics and population genetics have opened up new avenues enabling the understanding of underlying trait architectures thus expanding the possibilities for gene editing. In many cases, basic research in model systems can also help elucidate the effects of individual gene functions, even for complex gene families, however, applying that basic knowledge to crops of interest is required to fully validate that the targeted gene edits translate from model systems into crops. Based on the peer-reviewed literature, the sections that follow discuss the current view of gene editing for basic research and for trait development in Brassica napus in several impact areas.

Herbicide tolerance In the developed world, most growers deploy weed control systems to maximize yield by reducing or preventing weed competition for space, nutrients, water, and light. In the peer-reviewed scientific literature, there are many mutations described for various gene targets that confer resistance to a variety of herbicide chemistries. Amino acid substitutions at position P197 in ACETOHYDROXY ACID SYNTHASE (AHAS) paralogs, based on amino acid positions of the Arabidopsis thaliana protein (At3g48560), confer resistance to AHAS-inhibiting herbicides such as tribenuronmethyl in various plants (Chen et al. 2017). Editing of homologs of AHAS genes has been performed in several plant species, including Arabidopsis, corn, rice, and tobacco (Beetham et al. 1999; Zhu et al. 1999; Zhu et al. 2000; Kochevenko and Willmitzer 2003; Okuzaki and Toriyama 2004). More recently, several groups have also performed AHAS editing experiments using a base editing approach (Mishra et al. 2020; Wu et al. 2020a; Cheng et al. 2021). This methodology has emerged as an alternative tool to make nucleotide substitutions in a programmable manner without requiring a double strand break and has been deployed in a variety of plants including Arabidopsis, cotton, maize, rice, tomato, and wheat (Komor et al. 2016; Mao et al. 2019; Mishra et al. 2020). Cytidine base editor $(\mathrm{CBE})$ and adenine base editor $(\mathrm{ABE})$ are two versions currently in use, with ABEs developed through directed evolution of $E$. coli TadA (Gaudelli et al. 2017; Mishra et al. 2020). The methodology involves a Cas9 nickase (nCas9) or catalytically dead Cas protein (dCas9) fused to cytidine deaminase or adenosine deaminase that catalyze the deamination of cytosine (C) to uracil (U) or adenine to inosine (I), respectively. Uracil is recognized as thymine (T) and inosine recognized as guanine $(\mathrm{G})$ during replication allowing for base changes in daughter cells. Wu et al. (2020a) used a CRISPR/Cas9mediated CBE approach to attempt to edit three of the AHAS gene targets in canola, BnAHAS1 (BnaC01g25380D), BnAHAS2, and BnAHAS3 (BnaA01g20380D). A CBE construct containing the rat cytidine-deaminase (rAPOBEC1) was transformed into canola cv. J9712 hypocotyls using Agrobacterium tumefaciens (Wu et al. 2020a), generating 230 independent $\mathrm{T}_{0}$ plants. Of these putative transformants, 217 (94\%) were PCR positive using vector-specific primers. Of these, amplicons within the target region of each BnAHAS locus were sequenced revealing no lines had the expected transitions in BnAHAS2 or BnAHAS3 and 7 plants had edits in the BnAHAS1 gene target. The edited BnAHAS1 alleles consisted of 4 lines showing an intended $\mathrm{C}$ to $\mathrm{T}$ transition at amino acid position 197 with the remaining 3 lines displaying (11, 11, and $12 \mathrm{bp})$ deletions. Three of the 4 lines with edits in BnAHAS1 had an amino acid substitution P197S (CCT to TCT) with the fourth line having an amino acid substitution P197F (CCT to TTT). When sprayed with the AHAS inhibiting herbicide tribenuron, plants homozygous for the P197S allele were more tolerant than those heterozygous for this allele with the WT being severely damaged (Wu et al. 2020b). Amino acid substitutions at this position, observed in Arabidopsis and in a number of several weed species, confer resistance to various AHAS-inhibiting herbicides including tribenuron-methyl (Chen et al. 2017).

Cheng et al. (2021) also used a CBE that combined CRISPR-Cas9 nickase with a different cytidine deaminase APOBEC1 and uracil glycosylase inhibitor to introduce targeted C-to-T transition mutations near the PAM site of BnAHAS1 (BnaC01g25380D) and BnAHAS 3 (BnaA01g20380D). Based on the method of Liu et al. (2014), the authors accomplished this by hypocotyl transformation of the semi-winter OSR cv. Zhongshuang 6 using Agrobacterium tumefaciens. Of the 38 transgenic plants targeting these loci, 9 plants had edits in the BnaA01g20380D locus, and 8 plants had edits in the BnaC01g25380D locus. Of the mutations that were shown, a CCT to TTT codon substitution encoding a P197F amino acid substitution was observed in 2 plants for each of the BnAHAS1 and BnAHAS3 loci. Plants with homozygous mutations in both BnAHAS1 and BnAHAS3 were more tolerant to tribenuron than those with homozygous mutations in each AHAS locus (Cheng et al. 2021). While base editing methods have been shown effective in certain scope, substitutions arise in a narrow mutagenesis window of $\sim 4$ to 9 bases, in which multiple transitions may occur depending on the sequence 
context (Mishra et al. 2020). This can result in multiple inaccurate editing outcomes.

Flowering, plant hormones, and architecture-Flowering To coordinate the vegetative to floral transition, plants need to achieve the developmental competence to respond to environmental factors such as day length (photoperiod), and winter temperature (vernalization), as well as integrate endogenous signals including phytohormones (e.g., gibberellins) and sugars. In higher plants, flowering time and floral meristem identity are regulated by members of the phosphatidylethanolamine-binding protein (PEBP) family including FLOWERING LOCUS T (FT), a floral activator, and TERMINAL FLOWER 1 (TFL1), a floral repressor (Kardailsky et al. 1999; Kim et al. 2013). Five BnaTFL1 gene copies were identified in the genome of canola (Sriboon et al. 2020). Previously, mutations in BnaA10.TFL1 were shown not to have a significant effect on flowering time (Guo et al. 2014). Sriboon et al. (2020) used hypocotyl transformation of $\mathrm{cv}$. Westar using Agrobacterium tumefaciens with the CRISPR/Cas9 vector having guide RNA designed to target LOF mutations in the 5 BnaTFL1 loci BnaA02G0014100ZS, BnaA 10G0288700ZS, BnaC02G0013900ZS, BnaC03G001 6500ZS, and BnaC09G0608000ZS). Using the Zhang et al. (2015) protocol, callus lines of canola cv. Westar were transformed with two sets of constructs, each containing two gRNAs. Of 231 transgenic lines isolated, 100 were CRISPR/Cas9 PCR positive with 18 having at least one edited BnaTFL1 locus (Sriboon et al. 2020). The BnaC03.TFL1 biallelic LOF mutant exhibited an early flowering phenotype, while the other LOF mutant lines for the other gene copies had similar flowering times as the WT (Sriboon et al. 2020). Furthermore, LOF mutants of individual Bnatfl1 loci displayed altered plant architecture including significant reductions in plant height, branch initiation height, branch number, silique number, number of seeds per silique, and number of siliques on the main inflorescence (Sriboon et al. 2020). Together, as Sriboon et al. (2020) suggest, these results indicate functional divergence within this gene family in canola.

Also implicated in the vegetative to floral transition, the Arabidopsis sdg8-2 mutant flowers early because the gene expression of both the MADS-box transcription factor SUPPRESSOR OF OVEREXPRESSION OF CO 1 (SOC1) and FT are derepressed (Jiang et al. 2018). In Arabidopsis, histone $\mathrm{H} 3$ lysine 4 trimethylation ( $\mathrm{H} 3 \mathrm{~K} 4 \mathrm{me} 3$ ) and $\mathrm{H} 3 \mathrm{~K} 36 \mathrm{me} 3$ of core histone tails are required for the activation of FLOWERING LOCUS C (FLC) expression (Pien et al. 2008; $\mathrm{Xu}$ et al. 2008). When the expression of FLC is repressed following sufficient vernalization, it acts upstream of the integrators of the vegetative to floral transition $-S O C$ and $F T$ (described above).
While in canola it is not unexpected to have one to many paralogs of a target gene arising from each of the A and C genomes, to date within the genome of sequenced plants, the SET DOMAIN GROUP 8 (SDG8) histone lysine methyltransferase had only been observed as a single-copy gene. This presented an opportunity to understand functional divergence with the BnaA07.SDG8 gene having a single newly identified NNC domain with two adjacent domains in the BnaC06.SDG8 gene (Jiang et al. 2018). Jiang et al. (2018) transformed B. napus cotyledons of cv. XiangYou15 using Agrobacterium tumefaciens with a CRISPR/Cas9 vector designed to make LOF mutations in the 2 BnaSDG8 genes (BnaA07.SDG8 (BnaA07g33460D) and BnaC06.SDG8 (BnaC06g38010D)). Of 24 independent transformants obtained, 20 were PCR positive for the sgRNA-Cas9 cassette of which 3 lines displayed putative LOF alleles in the BnSDG8 loci. One line had biallelic mutations in each BnaSDG8 locus. A locus with one of those alleles remaining in frame having a 3-bp deletion, the second line had biallelic mutations in BnaC06.SDG8, and the third line termed Bnasdg8-1 was homozygous for LOF alleles in each of BnaA07.SDG8 and BnaC06.SDG8 (Jiang et al. 2018). Compared to the WT control, flowering time of null segregants lacking the transgene showed that Bnasdg8-1 had a similar early flowering phenotype to its EMS-derived counterpart in Arabidopsis (sdg8-2). Paralleling how this mutant leads to early flowering in Arabidopsis (described above), the authors showed that in the Bnasdg8-1 mutant, global changes in H3K36me2/3 methylation were present and that expression of 8 of the $9 F L C$ paralogs in OSR were repressed as well as the expression of both BnaSOC1 and BnaFT were upregulated (Jiang et al. 2018).

Kang et al. (2018) transiently delivered to canola cv. Tammi protoplast plant-compatible ABE binary vectors to target precise A-to-G substitutions in the FT locus (TAT to $\mathrm{CAT}$ or $\mathrm{CAC}$ - antisense strand) generating a $\mathrm{Y} 85 \mathrm{H}$ amino acid substitution in the FT protein as well as a mis-spliced RNA transcript of the phytoene desaturase gene. In using Agrobacterium-mediated transformation of these constructs into Arabidopsis, transgenic plants with late-flowering and albino phenotypes were obtained (Kang et al. 2018). Interestingly, the authors found (1) that base editing was only seen in the $\mathrm{T}_{1}$ generation when the RPS5A construct was used and (2) that A-to-G conversion efficiencies were higher when $\mathrm{T}$ preceded a target $\mathrm{A}$ (Kang et al. 2018).

Li et al. (2018a) used CRISPR/Cas9 gene editing to develop a spectrum of LOF alleles early in the first exon of 5 BnaSPL3 paralogs (BnC03.SPL3 (BnaC03g18800D), BnA04.SPL3 (BnaA04g19840D), BnC04.SPL 3 (BnaC04g44230D), BnA05.SPL3 (BnaA05g09840D), and BnCnn.SPL3 (BnaCnng05200D)). The authors used hypocotyl transformation of the semi-winter OSR cv. Zhongshuang 6 using Agrobacterium tumefaciens with the CRISPR/Cas9 vector having a single-guide RNA (gRNA) designed to 
target all 5 loci (Li et al. 2018a). Using PAGE analysis of annealed SPL3 amplicons, five plants exhibiting BnaSPL3 LOF mutations were identified in a screen of 72 transgenic samples (Li et al. 2018a). Sequencing confirmed LOF mutations in each line. Bnspl3 mutants exhibited developmental delay phenotype in the first generation. BnaSPL3, similar to its orthologs in Arabidopsis, are implicated in the regulation of the vegetative to floral developmental phase transition (Jung et al. 2012). Like AtSPL3, BnaSPL3 induces the expression of BnaAP1 (BnaC06.AP1-1, BnaC06.AP12, BnaC07.AP1-1, and BnaC07.AP1-2) a key MADS-box transcription factor required for this phase transition (Yamaguchi et al. 2009).

Once the vegetative to floral transition is initiated, a cascade of floral homeotic genes are activated (Weigel and Meyerowitz 1994). Zhang et al. (2018) used gene editing to develop a spectrum of mutant alleles in canola for homologs of the floral homeotic gene APETALA2 (AP2), which in part underlies A function in dicot flowers. Canola has four $B n a A P 2$ genes, two derived from B. rapa and two from B. oleracea (Parkin et al. 1995; Snowdon et al. 2002). Zhang et al. (2018) used hypocotyl transformation of SOSR cv. Westar (assumed) using Agrobacterium tumefaciens with a CRISPR/Cas9 vector designed to make LOF mutations in the four OSR AP2 homologs (which the authors termed BnaA-AP2a, BnaA-AP2b, BnaC-AP2a, BnaC-AP2b). Of 33 transformants, 6 were confirmed to contain LOF alleles with the genotypes BnaA-AP2a (+1 bp), BnaA-AP2b $(+1$ and -1 bp), BnaC-AP2a ( -4 and -5 bp), and BnaC-AP2b (+1 bp) with some plants showing the typical weak carpeloid or sepal carpeloid phenotypes (Zhang et al. 2018). For each of the four BnaAP2 genes, these frameshift mutations led to truncated non-functional proteins. A resulting ap2 quadruple mutant exhibited the characteristic ap2 flower phenotype (first observed in Arabidopsis) with carpeloid sepals, missing petals, and a reduced number of stamens, while having a normal pistil (Jofuku et al. 1994; Zhang et al. 2018).

Finally, towards developing a multilocular silique in canola, Yang et al. (2018) used CRISPR/Cas9 gene editing to develop a spectrum of LOF alleles by targeting the first exon and the C-terminal conserved CLV3/ESR related (CLE) domain 2 of the secreted peptide CLAVATA3 (BnaCLV3; BnaA04.CLV3 (BnaA04g15710D) and BnaC04.CLV3 (BnaC04g38990D)) or in the $5^{\prime}$ portion of its related receptors CLV1 (BnaA07.CLV1 (BnaA07g32120D) and BnaC06.CLV1 (BnaC06g36500D)) and BnaCLV2 (BnaA02.CLV2 (BnaA02g12070D) and BnaC02.CLV2 (BnaC02g45200D)). The CLAVATA (CLV) pathway is functionally conserved in plants. Although BnaC02.CLV3 (BnaC02g15230D) is present in the WOSR cv. Darmor-bzh reference sequence in Genoscope, it is not present, confirmed by Southern blotting, in the semi-winter OSR cv. J9707 (Yang et al. 2018). Of 860 transgenic lines generated, PAGE analysis identified 51 Bnaclv3 mutant lines in nearly 500 samples, 47 Bnaclv1 mutant lines in 101 samples, and 21 Bnaclv2 mutant lines in 37 samples. Amplicons from 22 edited lines of BnaCLV3 were Sanger sequenced with 17 lines that were homozygous double mutants of Bnaclv3, 4 lines were homozygous LOF mutants in BnA04.clv3, and 1 line was a homozygous LOF mutant in BnC04.clv3 (Yang et al. 2018). A multilocular phenotype can be recovered only when homozygous LOF mutants are obtained in both Bnaclv3 loci. These mutant BnaCLV3 lines produced more leaves and multilocular siliques with $33 \%$ more seeds per silique and a $25 \%$ higher seed weight than the wild-type and single mutant plants, potentially contributing to increased seed production (Yang et al. 2018). Although a multilocular silique phenotype was also obtained for the Bnaclv1 and Bnaclv2 loci, it was unstable (Yang et al. 2018).

Together these mutations, affecting plant height, branching, flowering time, pod number, seeds per pod, and seed weight are increasing diversity that does not currently exist in the canola gene pool and might be leveraged to increase yield in this important oilseed crop.

Plant hormones Gibberellins (GAs) are a well-characterized family of complex diterpenoid plant hormones that regulate dormancy and germination, extension growth, flowering, and fruit development in plants. In Arabidopsis, gibberellindeficient dwarfs were discovered, including Atga4 (Koornneef and van der Veen 1980). The GA4 gene transcripts encode GA $3 \beta$-hydroxylase responsible for converting growth inactive $\mathrm{GAs}$ such as $\mathrm{GA}_{20}$ and $\mathrm{GA}_{9}$ into their growth active forms $\mathrm{GA}_{1}$ and $\mathrm{GA}_{4}$ (Cowling et al. 1998). The $R G A$ gene encodes a DELLA protein that negatively regulates GA signaling and plant growth in many plant species. By adding diversity to loci effecting gibberellin biosynthesis, both plant height (lodging resistance) and yield can be more tightly controlled.

In one of the first large-scale CRISPR/Cas9 mutagenesis papers in canola, Yang et al. (2017) set out to create LOF alleles in 12 genes from 4 gene families that regulate plant development, using a strategy that employed two sgRNAs for each gene to assure a high mutation rate. The gene targets included 4 paralogs of the Arabidopsis REPRESSOR OF GA1-3 (RGA; Silverstone et al. 1997; Silverstone et al. 1998) gene (BnaA9.RGA, BnaC9.RGA, BnaA6.RGA [DS-1], and BnaC7.RGA [DS-3]), 3 paralogs of the Arabidopsis FRUITFULL (FUL; BnaA9.FUL, BnaC2.FUL, and BnaC7.FUL), and 5 paralogs of Arabidopsis $D A 1$ and DA2 (DA means "large" in Chinese; BnaA6.DA1, and BnaC5.DA1 as well as BnaA2.DA2.1 (BnaA02g18880D), BnaA2.DA2.2 (BnaA02g18890D), and BnaC6.DA2; Yang et al. 2017). Since BnaA2.DA2.1 and BnaA2.DA2.2 are a highly related ( $>98 \%$ identity) through tandem duplication, they were considered a single locus named BnaA2.DA2 (Yang et al. 2017). 
Of $435 \mathrm{~T}_{0}$ transgenic lines generated, 218 were derived from constructs targeting the DELLA and TVHYNP domains within the protein BnaRGA paralogs, 115 targeted the BnaDA paralogs, and 102 targeted the BnaFUL paralogs with 53 bearing LOF mutations. Further, for all three targets, $40 \%$ of 67 lines assayed using $\mathrm{T} 7$ endonuclease I showed biallelic LOF edits (Yang et al. 2017). Of all the lines with indels in the target sites, one did not have the CRISPR/Cas9 reagents inserted randomly within the regenerated plant's genome (Yang et al. 2017).

Gibberellins and abscisic acid play antagonistic roles in developmental processes including germination, elongation growth, and response to drought. Focusing on the BnaA6.RGA [DS-1] paralog, it was determined that the expression of this gene was greatly induced by drought and abscisic acid (ABA; Wu et al. 2020b). A gain of function mutant of BnaA6.RGA, BnaA6.rga-D having a mutant DELLA motif, displayed enhanced drought tolerance, with its stomatal closure being hypersensitive to ABA treatment whereas the quadruple LOF Bnarga mutant was less drought tolerant and was also less sensitive to ABA treatment ( $\mathrm{Wu}$ et al. 2020b). The Bnarga single mutants failed to show a difference in drought tolerance compared to the WT control $\mathrm{cv}$. Westar inferring that these genes are functionally redundant in this process (Wu et al. 2020b).

Targeting the conserved TVHYNP domain of 2 BnaRGA paralogs (BnaA09g18700D and BnaC09g52270D) in the semi-winter OSR cv. Zhongshuang 6, Cheng et al. (2021as described in the Herbicide section above) employed a CBE to introduce $\mathrm{C}$-to- $\mathrm{T}$ or $\mathrm{G}$-to-A transition mutations in the editing window. Of 63 transgenic plants targeting these loci, 2 plants had a CCG to CTG codon substitution encoding a P94L amino acid substitution in the BnaA09g18700D locus. Additionally, due to G-to-T and T-to-C base conversion, 16 plants had a CCG to TTG codon substitution encoding a P94L

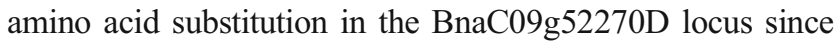
the gRNA perfectly matched the sequence of this locus. As expected, semi-dwarf mutants generated in this approach phenocopy the OSR ds-3 mutant (Zhao et al. 2017).

Cheng et al. (2021 - as described above) also used a $\mathrm{CBE}$ to target $\mathrm{C}$-to-T or G-to-A transition mutations in the conserved motif (GWPPV) of BnaIAA7 paralogs (BnaC01g43640D, BnaA03g36950D, BnaA05g16680D, and BnaC05g29300D) in the semi-winter OSR cv. Zhongshuang 6. Of 32 transgenic plants targeting these loci, 10 plants had transition mutations in the editing window for each of the BnaC01g43640D, BnaA03g36950D, and BnaA05g16680D loci, with 7 plants having edits in the BnaC05g29300D locus. Of the mutations that were shown for the BnaC05g29300D locus, all 4 plants had a CCT to TTT codon substitution encoding a P94L amino acid substitution (Cheng et al. 2021). Similar to the phenotype observed when the homolog of this gene is mutated in Arabidopsis, seedlings exhibit a dwarf phenotype with crinkled leaves and mature plants being extremely small and very late flowering.

Lin et al. (2018) also targeted LOF mutations in the GA4.a gene in canola protoplasts. The authors showed indels in a protoplast population that was treated with a plasmid expressing the CRISPR/Cas9 nuclease and the relevant gRNA, but did not regenerate plants from this population.

Unlike most of the transgenic work that preceded it, Tang et al. (2018) used Agrobacterium-mediated transformation of hypocotyls and visual selection of GFP positive plants in combination with antibiotic selection to identify LOF lines generated with CRISPR/Cas9 reagents. For their experiments with canola cv. Westar, more than $50 \%$ of the stable transformants had LOF mutations in the target genes (Tang et al. 2018). This visual selection system enabled LOF mutants to be obtained in all 4 canola AUXIN RESPONSE FACTOR2 (BnaA6.ARF2, BnaA9.ARF2, BnaC3.ARF2, and BnaC9.ARF2) loci, a repressor in auxin signaling (Schruff et al. 2006; Tang et al. 2018). Further, 5 GFP-positive plants were observed and confirmed to be PCR positive using Cas9-specific primers, with Sanger sequencing of the target regions used to confirm 1 to $3 \mathrm{LOF}$ alleles per target locus (Tang et al. 2018). Of these transgenic lines, 3 were quadruple mutants, 1 was a triple mutant (BnaA9.ARF2, BnaC9.ARF2, and BnaC3.ARF2), and the last line was double mutant (BnaC9.ARF2 and BnaC3.ARF2; Tang et al. 2018). Compared with the cv. Westar parent, the phenotypes of these Bnaarf2 mutants included shorter primary roots, a significantly increased seed size and 1000-seed weight, suggesting that, like its role in Arabidopsis, BnARF2 is a positive regulator of root elongation and a negative regulator of seed enlargement (Schruff et al. 2006; Tang et al. 2018).

Architecture Strigolactone, an emerging plant hormone that controls root growth, shoot branching, and interactions with symbiotic fungi and parasitic weeds, is a cleavage product of $\beta$-carotene (Xie et al. 2010). Stanic et al. (2020) generated CRISPR/Cas9-mediated LOF mutations in genes encoding the strigolactone receptor D14 in each of the A (LOC106435377) and C (LOC106431289) genomes following hypocotyl transformation of canola cv. Westar using Agrobacterium. Two primary transformants with a dwarf phenotype were obtained bearing biallelic LOF mutations in each of the 2 targeted loci (Stanic et al. 2020). Mean plant height was reduced 34\% resulting from a reduced internode length. These mutant plants also branched prolifically (200\% more than the cv. Westar parental control) and had 37\% more flowers leading to slight and non-significant increases of $12 \%$ and $10 \%$ in pod weight and seed yield, respectively (Stanic et al. 2020).

Zheng et al. (2020) also made LOF alleles in homologous gene targets that control lateral branching. A CRISPR/Cas9 approach was used to generate LOF mutations in genes 
encoding the 2 canola $B n a M A X 1$ homologs (BnaA03g22900D and BnaC03g26960D). MAX1 is an enzyme involved in strigolactone biosynthesis encoding a cytochrome P450 monooxygenase (CYP711A1) that converts carlactone to carlactonoic acid (Booker et al. 2005). The authors accomplished this by Agrobacterium-mediated transformation of the SOSR cv. 862 hypocotyls. Of the 4735 calluses obtained, 141 hygromycin-resistant positive transgenic plants were selected with 29 of these bearing LOF alleles in the target loci (Zheng et al. 2020). Unexpectedly, $3 \mathrm{~T}_{0}$ lines had large deletions ( $85-165 \mathrm{bp}$ ) causing them to lose the entire sgRNA target sites (Zheng et al. 2020). Of the 29 plants with LOF alleles, 4 lines bearing a diverse set of LOF alleles were taken to the next generation with some of these progeny being null segregants for the transgene. Plants with homozygous LOF alleles in both BnaMAX1 loci were $33 \%$ shorter, with a 2fold increased branching, having $33 \%$ more siliques, all of which contributed to a $25 \%$ increased yield per plant relative to the parental control (Zheng et al. 2020).

In summary, the work of Stanic et al. (2020) and Zheng et al. (2020) produced diversity in plant architecture that resulted in better mechanical harvest, reduced lodging, and increased yield.

Self-incompatibility Genetic systems for self-incompatibility (SI) are elaborate mechanisms that have evolved independently multiple times to promote outcrossing and maintain genetic diversity in many flowering plants. Each SI system prevents hydration, germination, and growth of self-incompatible pollen tubes. In the Brassicaceae, the SI system has two components encoded at the complex and polymorphic S-locus, (i) the transmembrane receptor $\mathrm{S}$ locus receptor kinase (SRK) expressed predominantly in the stigma and (ii) its ligand the $\mathrm{S}$ locus cysteine-rich (SCR) protein expressed in the tapetum that becomes part of the pollen coat (Nasrallah 1997; Schopfer et al. 1999; Suzuki et al. 1999). Commercially, SI systems can be used for developing hybrids. In Brassica napus, the Mlocus protein kinase (MLPK) BnaMLPKs has four paralogs, BnaA3.MLPK, BnaC3.MLPK, BnaA4.MLPK, and BnaC4.MLPK, which control SI (Chen et al. 2019). Agrobacterium-mediated transformation of CRISPR/Cas9 reagents was used to make loss of function alleles in each target gene, using the method of Yang et al. (2017), with two independent gRNAs per locus. In assessing 48 transgenic lines, 6 per gRNA per locus, 44 plants were determined to bear LOF alleles in one of the four target loci (Chen et al. 2019). Six of these plants were crossed to obtain two Bnamlpk quadruple mutants. Compared with the SI line S-70, the SI response was completely blocked in these quadruple mutants as evidenced by pollen germination, pollen tube elongation, and excellent seed set (Chen et al. 2019). By contrast, when pollen from the quadruple mutants was used to pollinate SI line S-70, no pollen germination or seed set was observed confirming that
BnaMLPKs are not the pollen SI determinants. Expression of six genes known to be associated with SI was assessed by qRT-PCR with three being differentially expressed in the Bnamlpk quadruple mutant compared to the SI line (Chen et al. 2019). Before pollination, the expression of the SRK and arm repeat containing 1 (ARC1), an E3 ubiquitin ligase, were suppressed in the Bnamlpk quadruple mutant disrupting SI signal transduction to enable pollen tube growth and elongation. Both MLPK and ARM1 are positive regulators that are known to interact with SRK in the SI pathway (Shi et al. 2016). By comparison, the self-compatible factor GLO1 was slightly induced in the Bnamlpk quadruple mutant (Chen et al. 2019). With or without pollination, 3 genes including thioredoxin h-like proteins THL1 and THL2 as well as Exo70A1 were not differentially expressed between the mutant and SI line S-70 (Chen et al. 2019). This work implicates the four BnaMLPK loci in the SI response in Brassica napus extending what was known from studying this response in Arabidopsis and Brassica oleracea.

Male sterility Hybrid systems enable increased plant vigor, disease resistance, and yield. The male sterility system 5 (MS5) gene underlies a three-line hybrid system in Brassica napus with the restorer allele being $\mathrm{MS5}^{\mathrm{a}}$, the male-sterile allele being $\mathrm{MS}^{\mathrm{b}}$ and the maintainer allele being $\mathrm{MS5}^{\mathrm{c}}(\mathrm{Lu}$ et al. 2012). This Brassica-specific nuclear localized gene encodes a protein containing conserved $\mathrm{N}$-terminal coiled coil (CC) and C-terminal DUF626 domains (Xin et al. 2016). When compared with MS5 ${ }^{\mathrm{a}}$, an 8-kb Mutator-like transposable element (MULE) occurs in the second intron of $\mathrm{MS}^{\mathrm{b}}$ (Xin et al. 2016). The MS5 ${ }^{\mathrm{a}}$ and $\mathrm{MS}^{\mathrm{c}}$ alleles are significantly divergent in both expression levels and amino acid sequence, displaying 18 amino acid differences over their more than 300 amino acid length (Xin et al., 2016). Towards understanding this triallelic sterility system, focusing on obtaining LOF alleles in the fertile lines cv. Westar $\left(\mathrm{MS5}^{\mathrm{c}} / \mathrm{MS}^{\mathrm{c}}\right)$ and $\mathrm{Y} 127$ (MS5 ${ }^{\mathrm{a}} \mathrm{MS5}^{\mathrm{a}}$ ), Xin et al. (2020) used Agrobacterium-mediated transformation CRISPR/Cas9 vectors with two gRNAs targeting the $\mathrm{CC}$ domain of this gene target, homozygous or biallelic ms 5 mutants from 5 of $8 \mathrm{~T}_{0}$ Y 127 plants and 2 of 20 $\mathrm{T}_{0}$ cv. Westar plants were male sterile (Xin et al. 2020). Compared with the WT, chromosomes were gathered as a compact mass in these engineered mutants at approximately the tetrad stage demonstrating that both $\mathrm{MS}^{\mathrm{a}}$ and $\mathrm{MS}^{\mathrm{c}}$ are necessary for male fertility by participating in the regulation of early meiosis in B. napus (Xin et al. 2020).

In a different, and more typical, two-line hybrid system, production of hybrid seed requires two lines, one line having a male sterile phenotype usually caused by a maternally inherited cytotoxic cytoplasmic male sterility (CMS) trait encoded in the mitochondrial genome, and the second line bearing a nuclear fertility restoring (Rf) gene (Iwabuchi et al. 1999). The mitochondrial gene orf125 is a CMS- 
associated gene in Kosena-type CMS (Iwabuchi et al. 1999). Using the method of Kohno-Murase et al. (1994), Ti plasmids expressing TALENs targeted to the mitochondria (termed mitoTALENs) were integrated into the nucleus of Kosenatype CMS Brassica napus L. (SW18) with transformants being regenerated under kanamycin selection (Kazama et al. 2019). Line SW18 is a cybrid that was originally created by asymmetric cell fusion between canola cv. Westar and a CMS radish (Raphanus sativus L. 'Kosena') and extensively backcrossed to cv. Westar to recover that parental phenotype (Sakai and Imamura 1992). Five transgenic lines were obtained, with PCR amplification confirming that 3 lines lacked the mitochondrial locus orf125 (Kazama et al. 2019). The fertility of all 3 lines lacking orf125 was restored confirming that this locus is responsible for the CMS phenotype in the Kosenatype CMS system (Kazama et al. 2019). This peer-reviewed publication demonstrates that gene editing can also be performed in the mitochondrial genome of Brassica napus.

Nutrition Canola is extremely sensitive to boron deficiency (Marschner 2012), preventing seed set even when flowering appears normal (Xu et al. 2002). Feng et al. (2020) used Agrobacterium-mediated hypocotyl transformation of canola $\mathrm{cv}$. Westar 10 using a CRISPR/Cas9 vector designed to make LOF mutations in the transcription factor BnaA9. WRKY47 (BnaA09g00350D). From 19 independent mutant lines, null segregants for 3 BnaA9.WRKY47 homozygous mutants were studied further. These lines had higher sensitivity to low boron content than WT plants demonstrating that BnaA9.WRKY47 contributes to the adaptation of canola to low boron stress (Feng et al. 2020). In part, this is likely since BnaA9.WRKY47 directly activates the expression of a boric acid channel NIP (nodulin 26-like intrinsic protein) BnaA3.NIP5; 1 and BnaC3.NIP5; 1 in roots under boron deficiency (Feng et al. 2020). This is reinforced by the fact that BnaA3.NIP5; 1 contains a conserved $\mathrm{W}$ box cis element in this downstream gene's promoter and that it is bound by BnaA9.WRKY47 in an electrophoretic mobility shift assay (Feng et al. 2020).

Disease resistance Disease resistance is a co-evolutional arms race between plant and pathogens. Even when suitable fungicides are available, a suitable application window may not be available and mutations in these rapidly evolving organisms may make them less effective. What's more, consumers are gravitating to organically produced foods and many governments are mandating the reduction of fungicides as part of "green deals" thus elevating a need for genetic disease resistance traits. Providing that yield and quality are not impacted, improving genetic disease resistance is therefore the preferred path.

Pathogens that can infect Brassica crops and cause production losses include viruses, bacteria, fungi, and oomycetes.
Yield, seed quality, and crop development are significantly impacted by pathogens of canola, which include the chytrid Plasmodiophora brassicae (club root); fungi including Leptosphaeria maculans (Blackleg or stem canker); the multinucleate filamentous pathogen Sclerotinia sclerotiorum (Sclerotinia stem rot or white mold), Alternaria brassicae (Alternaria Blight), Albugo candida (White Rust), Pseudocercosporella capsellae (White Leaf Spot), and Verticillium longisporum (Verticillium stem striping); the oomycete Hyaloperonospora parasitica (Downy Mildew); bacteria including Pseudomonas syringae (Blackrot); and viruses including the aphid transmitted Turnip Mosaic Virus (Murray and Brennan 2012).

Sclerotinia stem rot is a key disease in canola that can lead to poor oil quality and cause grain yield losses ranging from 10 to $80 \%$ (Purdy 1979). Infection of canola occurs when ascospores colonize senescent flower petals that have dropped onto leaves or petioles adjacent to the stem, followed by the development of necrotic lesions on the stem causing it to weaken or break and the plants to lodge and wilt. In canola, $B n W R K Y 11$ and BnWRKY70 genes are differentially expressed after inoculation with Sclerotinia sclerotiorum (Lib.) de Bary (Wu et al. 2016); in Arabidopsis, these single-copy loci are implicated in jasmonic acid (JA)- and salicylic acid (SA)-induced resistance to pathogens (Journot-Catalino et al. 2006; Hu et al. 2012). In canola, there are 6 paralogous loci that encode each of BnaWRKY11 (BnaA.WRKY11.a (BnaA03g51590D), BnaC.WRKY11.a (BnaC07g43320D), BnaA.WRKY11.b (BnaA01g34790D), BnaC.WRKY11.b (BnaC01g06900D), BnaA.WRKY11.c (BnaA08g12420D), BnaC.WRKY11.c (BnaC03g67520D)) and BnaWRKY70 (BnaA.WRKY70.a (BnaA07g165850D), BnaC.WRKY70.a (BnaC06g15910D), BnaA.WRKY70.b (BnaA09g35840D), BnaC.WRKY70.b (BnaC08g27340D), BnaA.WRKY70.c (BnaA04g02560D), BnaC.WRKY70.c (BnaCnng52600D)). Sun et al. (2018) used a CRISPR-Cas9 approach to inactivate the 2 BnaWRKY11 (BnaA.WRKY11.a (BnaA03g $51590 \mathrm{D}$ ) and BnaC.WRKY11.a (BnaC07g43320D)) and $4 B n W R K Y 70$ paralogs (BnaA.WRKY70.a (BnaA07g165850D), BnaC.WRKY70.a (BnaC06g15910D), BnaA.WRKY70.b (BnaA09g35840D), and BnaC.WRKY70.b (BnaC08g27340D)). These selected loci had the highest level of expression prior to inoculation, and following inoculation with Sclerotinia were most differentially expressed with the two BnaWRKY11 paralogs showing the greatest increase in expression, and the 4 BnaWRKY70 paralogs showing the greatest decrease in expression (Sun et al. 2018). Arising from transformation of hypocotyl segments of SOSR cv. J9712 using Agrobacterium, 30 PCR positive $\mathrm{T}_{0}$ transgenic plants with the NPTII selectable marker were obtained, 22 for the BnaWRKY11 target and 8 for the BnWRKY70 target. Of those, 12 lines had mutant alleles in BnaWRKY11 (2 different ones in BnaA.WRKY11.a with 23 in 
BnaC.WRKY11.a) and 4 lines had mutant alleles BnaWRKY70 (4 different ones in BnaA.WRKY70.a with 1 in $B n a A$.WRKY70.b). Given the gRNAs that were used, a deletion of 302 bp between the 2 gRNAs was observed in 1 of the edited BnaC.WRKY11.a lines (Sun et al. 2018). All edits in both BnaWRKY11 and BnaWRKY70 were indels with no substitutions observed (Sun et al. 2018). Editing chimeras were observed in two lines, 1 for each of BnaWRKY11 and BnaWRKY70 (Sun et al. 2018). Finally, for at least the BnaWRKY11 target, mutagenesis was not observed for some gRNAs in the $\mathrm{T}_{0}$ generation, but in at least 2 lines it was detected in the $\mathrm{T}_{1}$ generation (Sun et al. 2018).

The vast majority of Bnawrky 70 mutants showed editing in three paralogs of BnaWRKY70 in examined $\mathrm{T}_{1}$ plants. Based on the results of a detached leaf assay $48 \mathrm{~h}$ post-infection, homozygous triple mutants in Bnawrky70 (BnaA.wrky70.a, BnaA.wrky70.b, and BnaC.wrky70.b) showed enhanced resistance to Sclerotinia, while homozygous double mutants in BnaWRKY11 (BnaA.wrky11.a and BnaC.wrky11.a) failed to show a significant difference in resistance when compared to the parental check (Sun et al. 2018). By comparison, also based on the results of a detached leaf assay, transgenic plants that overexpressed BnaWRKY70 were more sensitive to this pathogen compared to the parental check (Sun et al. 2018). Taken together, these results show that LOF mutations in BnaWRKY70 might be used to achieve tolerance to Sclerotinia since this transcription factor functions as a negative regulator of Sclerotinia resistance in canola (Sun et al. 2018).

The hemibiotrophic fungal pathogen Verticillium longisporum is a soil-borne pathogen that infects the Brassicaceae causing stem striping (Depotter et al. 2016). Plants are infected when microsclerotia recognize root exudate from the host plant, germinate, and follow the nutrient gradient to reach the root. The fungus gains access to the plant by directly penetrating its epidermis or by taking advantage of wounds before its hyphae spread intra and intercellularly, ultimately producing conidia that are transported by the vascular system (Depotter et al. 2016). Eventually, this fungus begins its necrotrophic life phase in which it feeds on senescing leaves and stems.

In canola, transcriptome analysis showed a cohort of activated/upregulated genes 5 to $15 \mathrm{~d}$ following Verticillium (Vl43) infection (Pröbsting et al. 2020). Initially, T-DNA insertion LOF mutants for one of these genes, AtCRTla (Atlg56340), was identified in Arabidopsis. In this mutant, the ethylene signaling pathway is activated and is less susceptible to Vl43 infection (Pröbsting et al. 2020). Next, the mutagenesis approach termed Targeting Induced Local Lesions IN Genomes (TILLING) approach identified 10 mutations in the BnaCRTla paralog BnaA09.CRT1a (BnaA09g15400D), then using a CRISPR/Cas9 approach LOF alleles were generated in 2 BnCRTIa paralogs (paralogs BnaA09.CRTa (BnaA 09 g $15970 \mathrm{D}$ ) and BnaC09.CRT1a
(BnaC09g16150D)) - with the gRNA designed to discriminate against BnaA09.CRT1a paralogs (BnaA09g15970D and BnaC01g43040D; Pröbsting et al. 2020). The authors accomplished this by hypocotyl transformation of cv. Express using Agrobacterium tumefaciens (Pröbsting et al. 2020). Of 20 transformants, 4 lines had confirmed LOF mutations in BnaA09.CRTa (5 LOF alleles) and BnaC09.CRTla (12 LOF alleles). Three lines were biallelic in one BnaCRTla paralog and the fourth was biallelic in both paralogs. In the TILLING approach, one mutant line identified was hypersensitive to Vl43 infection with two others being more resistant. Without a timeconsuming backcross to clear the mutant line of mutations not associated with this phenotype, this result was inconclusive. In the CRISPR/Cas9 approach, the Bnacrta mutant plants confirmed that BnaA09.CRTa has a dominant role in a compatible interaction with this fungus and, therefore, when this locus bears homozygous LOF alleles, susceptibility to Vl43 is decreased (Pröbsting et al. 2020). As is the case in the Atcrta LOF mutant, ethylene signaling was also activated in the Bna A09.crt1 mutant. Together, in a crop with limited natural resistance to Verticillium, these results show a promising path towards achieving resistance to this devastating pathogen.

\section{Understanding host-pathogen interaction by pathogen} editing Globally, blackleg (Leptosphaeria maculans) is the most important disease affecting canola causing losses exceeding $\$ 900 \mathrm{M}$ annually (Fitt et al. 2008). There are numerous reports of resistance genes (mainly QTLs), but very few have been cloned and characterized for this pathogen. In specific gene for gene interactions of a pathogen with its host, there is a specific interaction of an avirulence (Avr) gene (e.g., UmAvr7) with its corresponding R gene (e.g., $R \operatorname{lm} 7$ ). In this example, the blackleg pathogen bearing $U m A v r 7$ is virulent on hosts that do not have the resistance gene $R \operatorname{lm} 7$. Not only can disease resistance be studied from the host (crop) side, but also by editing the pathogen.

In a disease survey conducted in western Canada, a total of 180 Avr gene races were identified from 964 isolates, with three major races observed: AvrLm-2-45-6-7, AvrLm2-4-5-67-S, and AvrLm-1-4-5-6-7-11-(S). The UMAvr7 isolate carried AvrLm1-2-3-4-9-11-LepR1LepR2-S-AvrLm5-6-7 and is PCR positive for AvrLm5, AvrLm6, and AvrLm4-7 (Zou et al. 2020). Previously, Parlange et al. (2009) determined that when codon358 in AvrLm4-7 has a transversion of " $\mathrm{C}$ " to "G", this pathogen is recognized by $R \operatorname{lm} 7$ and $R \operatorname{lm} 4 / 7$ in canola.

Zou et al. (2020) used Agrobacterium transformation of L. maculans isolate (DS103) (named UMAvr7) to make LOF alleles of the avirulence gene target AvrLm4-7 as previously described by Gardiner and Howlett (2004). The resulting mutant isolates were tested on different canola genotypes with 6 plants per genotype inoculated as described by Zhang et al. (2016). Among the mutant isolates, Mu3, 
hereafter termed as umavr 7 , when inoculated onto canola line 01-232-1 (Rlm7), developed large disease lesions similar to those observed in the susceptible cv. Westar, indicating a shift from avirulence to virulence. Further, by assessing $\mathrm{H}_{2} \mathrm{O}_{2}$ accumulation in cotyledons of resistant genotype 01-23-2-1, UMAvr7 caused limited accumulation around the wound site compared to mutant isolate umavr7 (Zou et al. 2020).

Recently, Jiquel et al. (2021), using a CRISPR-Cas9 editing approach in the blackleg pathogen and starting with a short list of 5 late effector candidates, were able to analyze the interaction of a blackleg ( $L$. maculans) late effector (avirulence) LmSTEE98 gene involved in the biotrophy to necrotrophy transition with RImSTEE98, a cognate resistance gene in canola that responds to this effector. Initially, narrowing down from a set of more than 200 canola candidate genotypes, it was determined that cv. Yudal elicited a hypersensitive resistance response to this effector. To validate this interaction, using the method described by Idnurm et al. (2017), CRISPR-Cas9 was used to make a panel of mutant alleles in LmSTEE98 in L. maculans. Four independent mutants with four different indels $(+1,-2,-9,-17 \mathrm{bp})$ were obtained and these putative LOF alleles were tested in a cotyledon stage model system assay to confirm that out-of-frame indels abolished this interaction, whereas the in-frame ( $-9 \mathrm{bp}$ ) deletion remained avirulent (Jiquel et al. 2021). Further, in a stem canker assay in B. napus $\mathrm{cv}$. Yudal, isolates bearing the three out-of-frame Lmstee 98 mutants elicited a stem necrosis resistance response, whereas the one bearing the in-frame ( -9 bp) deletion remained avirulent. All four isolates remained virulent in B. napus cv. Darmor-bzh, the susceptible check. With these tools in hand, the authors used a mapping approach using progeny from a dihaploid population developed from a cross of Darmor-bzh x Yudal to narrow the hypersensitive resistance response to a $350-\mathrm{kb}$ interval on chromosome A09 bearing the cognate resistance gene (Jiquel et al. 2021). This region contains 70 genes, based on the reference genome of the susceptible check cv. Darmor-bzh (Jiquel et al. 2021).

Li et al. (2018b) used a CRISPR/Cas9 approach aimed at achieving LOF alleles in the oxalate biosynthesis gene Ssoah1 GenBank accession number XM_001590428 in the necrotrophic pathogen Sclerotinia sclerotiorum (Lib.) de Bary (UF isolate) by delivering a plasmid encoding CRISPR/Cas9, gRNA expression cassette, and a hygromycin selectable marker. After three rounds of selection on potato dextrose agar (PDA) supplemented with the $\mathrm{pH}$ indicator bromophenol blue, the media surrounding strains that did not accumulate the chemical effector oxalic acid were also resistant to the selection agent hygromycin (Li et al. 2018b). Of a total of 92 hygromycin-resistant transformants, 38 (41\%) failed to acidify the growth media (Li et al. 2018b). In the parental mutant, rather than having small indels at the target site, large insertions, likely to be tandem insertion of the vector, disrupted the target gene (Li et al. 2018b). In meiosis to produce ascospores, tandem insertions undergo intrachromosomal recombination to reduce the tandem insertion to a single copy ( $\mathrm{Li}$ et al. 2018b). Like a previously described mutant $\Delta$ ss-oah1 in the 1980 strain (Liang et al. 2015), mutants lacking OAH1 function have enhanced production of compound appressorium, show decreased pigmentation on the surface of sclerotia, and in culture display a diffuse pattern of sclerotium development (Li et al. 2018b)

Seed-related gene editing targets-Yellow-seeded canola Zhai et al. (2020) using hypocotyl transformation of the semiwinter OSR cv. J9707 with Agrobacterium tumefaciens used CRISPR/Cas9 with guide RNA designed to target the basic helix-loop-helix (bHLH) transcription factors TRANSPARENT TESTA8 BnaA09.TT8 (BnaA09g22810D) and BnaC09.TT8b (BnaC09g24870D) to make a yellowseeded B. napus. A tandemly duplicated gene BnaC09.TT8a (BnaC09g24860D) lacked a critical conserved domain and did not appear to be transcribed. It was presumed to be a pseudogene and was not targeted. Transformation resulted in 333 PCR positive events (Zhai et al. 2020) producing a total of 48 targeted mutants identified by sequencing of the PCR products from the target sites (Zhai et al. 2020). Five plants, all determined to be homozygous double mutants, showed a visible LOF (yellow-seeded) phenotype, whereas the single homozygous mutants had the parental phenotype confirming that these two genes function redundantly (Zhai et al. 2020). Homozygous mutations at the target sites within BnaTT8, mainly due to frame shifts, were determined to cause non-functional proteins (Zhai et al. 2020). By blocking the proanthocyanidin-specific deposition in the inner layer of the seed coat, the desired phenotype was obtained in lines bearing LOF mutations in both BnaTT8 functional copies with this double mutant having elevated seed oil, likely resulting from its thinner seed coat, and protein content in addition to an altered fatty acid (FA) composition (Zhai et al. 2020). In B. rapa and B. juncea, natural LOF mutants in these genes lead to a yellow seeded trait ( $\mathrm{Li}$ et al. 2012a, 2012b; Padmaja et al. 2014).

Xie et al. (2020) used hypocotyl transformation of cv. J9712 with Agrobacterium tumefaciens, using CRISPR/Cas9 with gRNAs designed to target LOF alleles in the R2 and R3 domain of the Myb123 transcription factors BnaA08.TT2 (BnaA08g29930D) and BnaC08.TT2 (BnaC08g07960D) towards achieving a yellow-seeded canola. Following selection, 81 transformants had PCR products from the target sites sequenced identifying a total of 4 plants with targeted mutant alleles, three alleles for each of BnaA08.TT2 and BnaC08.TT2 (Xie et al. 2020). In the $\mathrm{T}_{0}$ generation, 2 plants had a mutant allele in either BnaA08.TT2 or BnaC08.TT2 and the other two 
with biallelic mutations in either BnaA08.TT2 or BnaC08.TT2 and hemizygous mutations in the other allele; no homozygous mutant lines were obtained (Xie et al. 2020). As was the case for BnaTT8, homozygous BnaTT2 double mutants were yellow-seeded due to significantly reduced flavonoid content, especially epicatechin and isorhamnetin, increased oil content and improved fatty acid composition with more polyunsaturated fatty acids (Xie et al. 2020). Yellow seeds with homozygous BnaTT2 double LOF mutants also had reduced lignin and synaptic acid (Xie et al. 2020).

Reduced phytate Phytic acid is the principle stored form of phosphorus in plants but is considered anti-nutritive for monogastric animals including humans because it chelates minerals that impede their absorption. This is due to phytases in these animals being unable to hydrolyze it to free inorganic phosphate and myo-inositol. Further, phytic acid, which accounts for 2-5\% of canola grain (Sashidhar et al. 2020), if left undigested,, passes through the gastrointestinal tract causing environmental problems including eutrophication that threatens aquatic life. Globally, more than 400 dead zones caused by eutrophication exist. Inositol tetrakisphosphate kinase (ITPK) catalyzes the penultimate step in the synthesis of phytic acid in plants (Raboy 2009). Sashidhar et al. (2020) used a CRISPRCas9 approach to inactivate the 15 paralogs in the canola ITPK gene families: BnaITPK1 (BnaA03.ITPK1 (BnaA03g06170D), BnaC03.ITPK1 (BnaC03g07940D), BnaA10.ITPK1 (BnaA10g17710D), and BnaC09.ITPK1 (BnaC09g41080D)), BnaITPK2 (BnaA01.ITPK2 (BnaA01g03220D), BnaC01.ITPK2 (BnaC01g04480D), BnaA03.ITPK2 (BnaA03g50630D), BnaC03.ITPK2 (BnaC03g66400D), BnaAnn.ITPK2 (BnaAnng34680D), and BnaC07.ITPK2 (BnaC07g44490D)), BnaITPK3 (BnaA03.ITPK3 (BnaA03g38640D) and BnaCnn.ITPK3 (BnaCnng47190D)), and BnITPK4 (BnaA05.ITPK4 (BnaA05g03660D), BnaC04.ITPK4a (BnaC04g03240D), and BnaC04.ITPK4b (BnaC04g03250D)). After Agrobacterium transformation of 321 hypocotyl segments of the SOSR cv. Haydn and selecting for the transgene, 23 shoots from 10 independent transgenic events, all PCR positive for Cas 9 as part of the transgene, were regenerated to whole plants (Sashidhar et al. 2020). Each independent transgenic line had LOF alleles in up to 4 gene families (Sashidhar et al. 2020). Further analysis of 3 lines using Sanger sequencing of PCR amplicons revealed 3 to 8 mutant alleles per locus except BnaA03.ITPK1 and BnaC03.ITPK1, for which no edited alleles were observed (Sashidhar et al. 2020). At the $T_{4}$ generation, 2 triple LOF locus mutants (in BnaA10.ITPK1, BnaC09.ITPK1, and BnaC04.ITPK4 b) exhibited low phytic acid (with a 30\% decrease) and a concomitant increase (approximate doubling) of free phosphorus (Sashidhar et al. 2020). This work describes an important advance towards improving meal quality in canola.
Oil seed content Using a combination of chemical mutagenesis and transformation of hypocotyl segments of WOSR cv. RS306 using Agrobacterium tumefaciens containing the CRISPR/Cas9 reagents, Karunarathna et al. (2020) aimed to increase seed oil content (and thus oil per acre) by producing LOF alleles in the SEED FATTY ACID REDUCER genes BnSFAR4 (BnaA06.SFAR4a, BnaA06.SFAR4b, BnaC03.SFAR4 a, and BnaCxx.SFAR4.b) and BnSFAR5 (BnaA03.SFAR5 and BnaC07.SFAR5)-from the GDSL lipases. To date, about 1100 GDSL lipases/ esterases have been found in plants with this family consisting of 105 members in the model plant Arabidopsis (Lai et al. 2017). Eight hundred fifty-seven, 442 , and 754 excised hypocotyls were transformed to achieve LOF alleles in BnaSFAR1, BnaSFAR4, and BnaSFAR5 yielding, 2, 5, and 2 transgenic plants, respectively (Karunarathna et al. 2020). For BnaSFAR4, 13 different mutant alleles with single nucleotide insertions and deletions were made with each mutation producing a premature stop codon leading to a truncated protein (Karunarathna et al. 2020). Mutant segregation was Mendelian. The authors attempted to segregate away the transgene and were successful for BnaSFAR4, but not for BnaSFAR5, which was assumed to be due to a complex insertion pattern (Karunarathna et al. 2020). Interestingly, the Bnasfar4 mutant lines showed significantly larger oil bodies (Karunarathna et al. 2020). Seed oil content increased late in the seed maturation phase by making LOF mutations in Bnasfar4 (9.7-14.5\%) and Bnasfar5 $(<10 \%)$ genes without adversely affecting seed germination or vigor (Karunarathna et al. 2020).

Targeting the largest number of homologous loci to date, Zhang et al. (2019) used hypocotyl transformation of the semi-winter OSR cv. J2016 using Agrobacterium tumefaciens with the CRISPR/Cas9 vector having 3 guide RNAs designed to target conserved regions in the 7 homologous BnaLPAT2 genes (2 BnaA07.LPAT2 loci, BnaC07.LPAT2, BnaA09.LPAT2, BnaC08.LPAT2, BnaA04.LPAT2, $B n a C 04 . L P A T 2$ ) and a single-guide RNA designed to target a conserved region in the 4 homologous BnaLPAT5 genes (BnaA05.LPAT5-, BnaC05.LPAT5-, BnaC01.LPAT5-, and BnaUnk.LPAT5-). Respectively, the BnaLPAT2 and BnaLPAT5 families were $60.0 \%$ and almost $80 \%$ identical suggesting that their functions in oil biosynthesis have diverged (Zhang et al. 2019). Of 247 regenerated plantlets, 227 were PCR positive for the transgene, with target region sequencing revealing 100 bearing LOF alleles in each of the 7 BnaLPAT2 and 4 BnaLPAT5 gene targets. As would be expected, when multiple gRNAs were combined on a single construct, deletions of the regions between the target sites were obtained (Zhang et al. 2019). No off-target editing of 14 potential off-target sites with 1,2 , or 3 mismatches was detected (Zhang et al. 2019). Phenotypically, seeds with mutant Bnalpat2 and Bnalpat5 genes had a decreased oil content 
(24-39\%), displayed a wrinkled phenotype, and had fewer larger oil bodies perhaps as a result of smaller oil bodies coalescing; protein bodies were affected, and starch accumulated in mature seeds (Zhang et al. 2019). Specifically, when the BnaLPAT2 targets were disrupted, on average C18:0 and C20:0 increased $60 \%$ and $90 \%$, respectively, whereas C18:1, $\mathrm{C} 18: 2$, and $\mathrm{C} 18: 3$ decreased on average $8 \%, 8 \%$, and $21 \%$, respectively.

High oleic oil Compared with other vegetable oils, canola oil is high in unsaturated fatty acids comprised of monounsaturated oleic acid and an optimal 2:1 ratio of the polyunsaturated linoleic and linolenic acid (Hu et al. 2006). Vegetable oils with higher oleic acid (C18:1) contents are desirable because of their higher thermal stability and improved shelf life. This can be important for applications in both food and industrial applications. In plants, the stearoyl-acyl carrier protein desaturase encoded by the FATTY ACID DESATURASE 2 $(F A D 2)$ genes catalyzes desaturation of stearic acid (C18:0) to oleic acid (C18:1). The B. napus genome contains four $F A D 2$ orthologues with two genes originating from $B$. rapa (BnaA05.FAD2a (BnaA05g26900D) and BnaA01.FAD2b (BnaA01g09250D)) and the other two from $B$. oleracea (BnaC05.FAD2a (BnaC05g40970D) and BnaC01.FAD2b; Yang et al. 2012). Three copies (BnaA05.FAD2a, BnaC05.FAD2a, and Bna05C.FAD2b) appear intact and likely to be functionally redundant, in contrast to BnaA01.FAD2b, which appears to be a non-functional pseudogene resulting from a single bp deletion at nucleotide 164 that leads to a premature stop codon at position 411 (Yang et al. 2012; Lee et al. 2013; Well et al. 2014; Bai et al. 2019; Huang et al. 2020).

Okuzaki et al. (2018) used hypocotyl transformation of cv. Westar using Agrobacterium tumefaciens with the CRISPR/ Cas9 vector designed to target BnaFAD2a in both the A and $\mathrm{C}$ genomes. Out of $20 \mathrm{~T}_{0}$ transgenic plants obtained, three had LOF alleles within the gene target with only two transmitting the LOF allele to their progeny (Okuzaki et al. 2018). One of these plants was backcrossed to the parent $\mathrm{cv}$. Westar to generate 3 null segregants for the transgene (containing the selectable marker and CRISPR/Cas9 reagents) from the target LOF BnaFAD2 allele (4-bp deletion; Okuzaki et al. 2018). In grain from the homozygous mutant plants, oleic acid content increased significantly and the $\mathrm{C} 18$ polyunsaturated fatty acids significantly decreased (Okuzaki et al. 2018). Recently, Huang et al. (2020) used hypocotyl transformation of cv. J9707, a semi-winter B. napus, with Agrobacterium tumefaciens to edit all three active BnaFAD2 gene targets (Zhou et al. 2002). Transformation resulted in 108 PCR positive events (Huang et al. 2020). Of these, 16 yielded LOF BnaFAD2 alleles; 3 were identified in BnaC05.FAD2a, 2 in BnaA01.FAD2b with the remaining 11 being in BnaA05.FAD2a, and no edits in BnaC01.FAD2b (Huang et al. 2020). Further, of the 11
BnaA05.FAD2a mutant lines, 6 had edits in both alleles (biallelic). Finally, two lines were heterozygous for mutations in each of BnaC05.FAD2a and BnaA05.FAD2a with two lines being heterozygous for mutations in each of BnaA01.FAD $2 b$ and with BnaA05.FAD2a being heterozygous in one line and having biallelic edits in the other (Huang et al. 2020). Off-target sites were assessed with none detected - all of which had 4 or 5 mismatches (Huang et al. 2020). Similar to what was observed by Okuzaki et al. (2018), in grain from the homozygous BnaA05.fad2a mutant plants, oleic acid content increased significantly $(6-16 \%)$ and the $\mathrm{C} 18$ polyunsaturated fatty acids significantly decreased (Huang et al. 2020). In grain from the homozygous BnaC05.fad2a mutant plants, oleic acid content increased somewhat (about 5\%) and the $\mathrm{C} 18$ polyunsaturated fatty acids decreased by a similar magnitude (Huang et al. 2020). Assessed as single seeds for a segregating population, an additive effect was observed when homozygous LOF mutations were combined for both BnaA05. fad $2 a$ and BnaC05.fad2a (Huang et al. 2020). In this population, 2 single seeds showed an oleic acid content exceeding $>85 \%$ (Huang et al. 2020). Unlike Okuzaki et al. (2018), the reagent bearing transgene was not segregated away; therefore, mutations in the target genes in the progeny continued to accumulate (Huang et al. 2020).

Pod shatter reduction Seed pod opening and dispersal at harvest significantly affects yields with typical losses of $8-12 \%$ increasing upwards to 50\% (Kadkol et al. 1986; Jaradat et al. 2014), if optimum harvest timing and practices, such as swathing, are not employed. A pod shatter reduction (PSR) trait offers farmers the advantages of flexibility for when to harvest the mature crop, reducing dockage for green seed (Hu et al. 2015), and peace of mind that grain will not be easily lost due to adverse weather, as well as savings in both time and fuel afforded from one less pass across the field. Additionally, more effective weed and disease control is provided since shattered grain can remain viable in soil for up to $17 \mathrm{yr}$, becoming weeds (volunteer plants) in the subsequent crops (Morgan et al. 2000; Jørgensen et al. 2007).

Liljegren et al. (2004) and Raman et al. (2014) investigated the genetic basis for pod shatter reduction and suggested a cascade of transcription factors was central to the phenotype. Using information related to pod structure elucidated through comparisons to Arabidopsis, it was determined that genes encoding two MADS-box transcription factors SHATTERPROOF (SHP1) and SHATTERPROOF2 (SHP2), in addition two basic helix-loop-helix (bHLH) transcription factors INDEHISCENT (IND) and ALCATRAZ (ALC), influence shatter. In Arabidopsis, the SHP proteins induce the expression IND, ALC, and SPATULA (SPT) (Ferrándiz et al. 2000; Liljegren et al. 2000). Members of the Brassicaceae including B. napus and Arabidopsis have a seed pod (fruit) with two carpel valves joined to a replum, a septum that 
divides the two carpels. A layer of two to three parenchymous cells between the valve edges and the replum forms the separation or dehiscence zone and can be identified because adjacent replum tissue contains the main vascular elements of the pod. Cell wall-degrading enzymes including cellulases and polygalaturanases are expressed in the dehiscence zone leading to its formation. While the seed pods are drying, they split along the dehiscence zone to shatter and release seed. Indehiscent mutants are completely indehiscent due to the absence of both lignified cells and the separation layer at the predetermined breaking point of the silique (Liljegren et al. 2004); alc mutants, however, only lack the separation layer (Rajani and Sundaresan 2001). Clearly, shatter resistance is a quantitative trait resulting from variation at multiple loci (Raman et al. 2014; Liu et al. 2016).

Braatz et al. (2017) used a CRISPR-Cas9 approach to inactivate the two BnaALC homologs, BnaA07.ALCa (BnaA07g12110D) and BnaC07.ALCa (BnaC07g16290D), upstream of the DNA-binding basic helix-loop-helix (bHLH) in canola. Arising from Agrobacterium-mediated transformation of 625 hypocotyl segments of SOSR cv. Haydn, over a period of 9-11 mo, 70 calluses were obtained of which 112 regenerated shoots with 4 bearing LOF frame shift alleles, two in each BnaALC homolog. When plants bearing the mutant BnaALC loci were assessed for shatter resistance, improvement of this phenotype was marginal, owing to high shatter resistance of the transformed cultivar itself (Braatz et al. 2018). Previously, this transformation method had most commonly been employed with the Westar cultivar (De Block et al. 1989).

Like Braatz et al. (2017), Zhai et al. (2019) used a CRISPR/Cas9 approach to independently inactivate the two BnaIND homologs, BnaA03.IND (BnaA03g27180D) and BnaC03.IND (BnaC03g32180D) as well as the two BnaALC homologs (described above). The authors accomplished this by hypocotyl transformation of the semi-winter OSR cv. J9707 using Agrobacterium tumefaciens (Zhou et al. 2002; Zhai et al. 2019). Of the more than 250 transformants for each of the two targets, $>75 \%$ were PCR positive for the intended transgene (Zhai et al. 2019). Further characterization of these PCR positive transgenic lines identified 14 with confirmed LOF alleles in either $B n a I N D$ or BnaALC, including one single mutant BnaA03.ind, two BnaC03.ind single mutants, eight Bnaind double mutants, and three Bnaalc double mutants (Zhai et al. 2019). With the exception of one Bnaind mutant line, the remaining lines had loci with frameshifts leading to nonfunctional proteins. Similar to what was observed by Braatz et al. (2017), the PSR phenotype of the homozygous double mutant Bnaalc lines was similar to that of the parental control (Zhai et al. 2019). All homozygous double mutant Bnaind lines barely opened after their seeds matured and dried. For pod shatter resistance, these genes have partially redundant functions in canola pods with BnaA03.IND being more highly expressed and responsible for more of this quantitative effect than BnaC03.IND (Zhai et al. 2019). Developed using chemical mutagenesis, the dominant commercial pod shatter resistance trait in the North American market affects the BnaIND homologs.

Based on its function in Arabidopsis, the JAGGED $(A t J A G)$ gene encodes a protein with a single $\mathrm{C} 2 \mathrm{H} 2$ zincfinger domain that controls the development of lateral organs (Dinneny et al. 2004). Zaman et al. (2019), with the aim of reducing pod shatter, used a CRISPR/Cas9 approach to generate LOF mutations in genes encoding the 5 BnaJAG paralogs (BnaA02.JAG (BnaA02g13870D), BnaC02.JAG (BnaC02g18270D), BnaC06.JAG (BnaC06g30050D), Bna A07.JAG (BnaA07g27150D), and Bna A08.JAG (BnaA08g24290D)). The authors used hypocotyl transformation of the semi-winter OSR cv. Zhongshuang 6 using Agrobacterium tumefaciens with the CRISPR/Cas9 vector having gRNAs designed to target all 5 loci and a separate construct targeting the BnaA08.JAG locus (Zaman et al. 2019). From plants regenerated from 106 callus lines, 41 were confirmed to be PCR positive for the NPTII selection marker gene. PAGE of annealed JAG amplicons identified 25 plants bearing at least one mutated paralog. Among these $25 \mathrm{mu}-$ tants, 6 plants appeared to carry mutant alleles for all $5 J A G$ paralogs (Zaman et al. 2019). Line BnaJAG-5 was Sanger sequenced to confirm LOF loci in all 5 paralogs (Zaman et al. 2019). All 6 presumed pentuple mutants had a similar phenotype with pods having a cylindrical body that developed around the transmitting tract cells that guide the pollen tube from the stigma to the ovary (Zaman et al. 2019). The ovary appeared to be comprised of a number of undifferentiated cells (likely callus), without distinct differentiation of valves, replum, septum, and valve margins (Zaman et al. 2019). Pseudoseeds formed, but ultimately no viable seed was obtained from these lines. A separate line BnaJAG-33 was confirmed to have biallelic mutations in BnaA08g24290D (Zaman et al. 2019). This line appeared to be more resistant to pod shatter, with reduced lignification observed in the dehiscence zone; however, it had 50\% wider and shorter pods with twisting valves along its whole length and only $10 \%$ of the seeds per pod compared with the WT (Zaman et al. 2019).

\section{Conclusions}

With publications beginning about 4 yr ago, in laboratories scattered around the globe, an increasing number of loci within the Brassica napus genome have been gene-edited using various technologies and reagent delivery methods. These efforts and their resulting publications have furthered basic research and offered opportunities for trait development in this important oilseed crop. 
A few publications describe delivery of gene-editing reagents into protoplasts; however, plants bearing loss of function alleles developed in the papers herein cited were predominantly obtained using Agrobacterium-mediated transformation, principally of more easily transformed cultivars including Westar. Most applications of gene-editing in canola to date have involved delivery of DNA-based CRISPR/Cas9 editing reagents that are first integrated into the genome and expressed as a transgenic construct, and then segregated away by breeding as null segregants to leave only the desired LOF allele(s). However, this may be difficult to achieve in circumstances in which (1) DNA-based reagents may incorporate within the cut site or in a region tightly linked to the target allele or (2) in the case of complex multiplex/multitarget traits, in which large populations of at least $4^{\mathrm{n}}$ (where $\mathrm{n}$ is the number of gene-edited loci) are required to obtain null segregants with these traits. Further, while DNA-based editing reagents allow the ability to enrich for transformants using selective agents such as kanamycin, hygromycin, or BASTA $^{\mathrm{TM}}$, the time in culture required for efficient selection feeds a progressive gene-editing process that can lead to chimeras. For instance, Yang et al. (2017) observed that more than $30 \%$ of the transgenic $\mathrm{T}_{0}$ plants analyzed were chimeric. This progressive editing may occur at a low frequency during the growth of the transgenic plants, and even in subsequent generations where the remaining WT alleles can be edited (Yang et al. 2018). To truly accelerate plant breeding, development of truly genotype independent methods for geneediting within the range of Brassica napus cultivars is required.

A range of methods have been used to edit gene sequences in plants that have broadened the options to precisely fine-tune gene sequence including rare cutting endonucleases known as meganucleases, followed by engineered zinc finger nucleases, transcriptional activator-like effector nucleases (TALENs), and most recently, clustered regulatory interspaced short palindromic repeat (CRISPR)-associated protein (Cas) (CRISPR/Cas) - the latter which has become synonymous with the field of gene-editing (Chen and Gao 2014; van Eck 2020). Extending these editing tools, additional nucleases, such as Cas12a (Cpfl) with its differing PAM (protospacer adjacent motif) requirement compared to Cas9, have further expanded the available target sites for geneediting and new approaches, such as covalently linked activators, repressors, methylases, and base editors, as well as prime-editing, have broadened the options to precisely fine-tune gene sequence, including gene expression (Razzaq et al. 2019; Lin et al. 2020; van Eck 2020). As elaborated in this review article, most geneediting applications in Brassica napus have involved making LOF alleles in various gene targets. Base-editing was deployed for a few targets enabling single letter changes within a few bases of the PAM site. These yielded a subset of expected letter changes. Further and precisely augmenting diversity within the Brassica napus genome will be enabled by extending past CRISPR/Cas9 to other CRISPR-associated systems as well as the application of editing technologies such as homology-directed geneediting or prime-editing to enable the most precise and defined edits in this important oilseed crop.

As articulated throughout this review article, within the $B$. napus genome, many gene targets have at least one homo$\log$ from each of the $\mathrm{A}$ and $\mathrm{C}$ genomes with several targets, owing to the polyploidization events that led to the Brassicaceae being represented by 4 , and even up to 8 independent loci (Chalhoub et al. 2014). Many traits such as yield and disease tolerance are complex and expected to require edits in multiple independent loci. Multiplex genome editing involves the simultaneous delivery of reagents to precisely edit multiple, related or unrelated, loci within a single cell and therefore within a single regenerated plant. Going further, the next step in the evolution of gene- editing technologies will be to combine gene-edited traits de novo as would be the case through introgression breeding, but with dramatically shorter timelines.

Acknowledgements The author would like to thank all members of the Cibus team, current and past, for their many contributions to the exciting field of precision genome editing technologies in plants. The author is an employee of Cibus, an industry leader in developing and applying precision gene editing tools to meet agricultural, industrial, and human health needs. Further, the author would like to thank Noel Sauer, Christian Schöpke, Jerry Mozoruk, Tony Moran, Andrew Walker, Jim Radtke, Javier Narváez-Vásquez, Zhixia Niu, Peter Luginbühl, Steve Sanders, Amir Sattarzadeh, and Daniel Gobena for critical review of this review article.

Open Access This article is licensed under a Creative Commons Attribution 4.0 International License, which permits use, sharing, adaptation, distribution and reproduction in any medium or format, as long as you give appropriate credit to the original author(s) and the source, provide a link to the Creative Commons licence, and indicate if changes were made. The images or other third party material in this article are included in the article's Creative Commons licence, unless indicated otherwise in a credit line to the material. If material is not included in the article's Creative Commons licence and your intended use is not permitted by statutory regulation or exceeds the permitted use, you will need to obtain permission directly from the copyright holder. To view a copy of this licence, visit http://creativecommons.org/licenses/by/4.0/.

\section{References}

Bai S, Engelen S, Denolf P, Wallis JG, Lynch K, Bengtsson JD, Van Thournout M, Haesendonckx B, Browse J (2019) Identification, characterization and field testing of Brassica napus mutants producing high-oleic oils. Plant J 98:33-41

Beetham PR, Kipp PB, Sawycky XL, Arntzen CJ, May GD (1999) A tool for functional plant genomics: chimeric RNA/DNA 
oligonucleotides cause in vivo gene-specific mutations. Proc Natl Acad Sci USA 96:8774-8778

Booker J, Sieberer T, Wright W, Williamson L, Willett B, Stirnberg P, Turnbull C, Srinivasan M, Goddard P, Leyser O (2005) MAX1 encodes a cytochrome $\mathrm{P} 450$ family member that acts downstream of MAX3/4 to produce a carotenoid-derived branch-inhibiting hormone. Dev Cell 8:443-449

Braatz J, Harloff HJ, Mascher M, Stein N, Himmelbach A, Jung C (2017) CRISPR-Cas9 targeted mutagenesis leads to simultaneous modification of different homoeologous gene copies in polyploid oilseed rape (Brassica napus). Plant Physiol 174:935-942

Braatz J, Harloff HJ, Emrani N, Elisha C, Heepe L, Gorb SN, Jung C (2018) The effect of INDEHISCENT point mutations on silique shatter resistance in oilseed rape (Brassica napus). Theor Appl Genet 131:959-971

Chalhoub B, Denoeud F, Liu S, Parkin IA, Tang H, Wang X, Chiquet J, Belcram H, Tong C, Samans B, Corréa M (2014) Early allopolyploid evolution in the post-Neolithic Brassica napus oilseed genome. Science 345:950-953

Chen F, Yang Y, Li B, Liu Z, Khan F, Zhang T, Zhou G, Tu J, Shen J, Yi $\mathrm{B}, \mathrm{Fu} \mathrm{T}$ (2019) Functional analysis of M-locus protein kinase revealed a novel regulatory mechanism of self-incompatibility in Brassica napus L. Int J Mol Sci 20:3303

Chen K, Gao C (2014) Targeted genome modification technologies and their applications in crop improvements. Plant Cell Rep 33:575-583

Chen Y, Wang Z, Ni H, Xu Y, Chen Q, Jiang L (2017) CRISPR/Cas9mediated base-editing system efficiently generates gain-of-function mutations in Arabidopsis. Sci China Life Sci 60:520-523

Cheng H, Hao M, Ding B, Mei D, Wang W, Wang H, Zhou R, Liu J, Li C, Hu Q (2021) Base editing with high efficiency in allotetraploid oilseed rape by A3A-PBE system. Plant Biotechnol J 19:87-97

Cowling RJ, Kamiya Y, Seto H, Harberd NP (1998) Gibberellin doseresponse regulation of GA4 gene transcript levels in Arabidopsis. Plant Physiol 117:1195-1203

De Block M, De Brouwer D, Tenning P (1989) Transformation of Brassica napus and Brassica oleracea using Agrobacterium tumefaciens and the expression of the bar and neo genes in the transgenic plants. Plant Physiol 91:694-701

Depotter JR, Deketelaere S, Inderbitzin P, Tiedemann AV, Höfte M, Subbarao KV, Wood TA, Thomma BP (2016) Verticillium longisporum, the invisible threat to oilseed rape and other brassicaceous plant hosts. Mol Plant Pathol 17:1004-1016

Dinneny JR, Yadegari R, Fischer RL, Yanofsky MF, Weigel D (2004) The role of JAGGED in shaping lateral organs. Development 131: 1101-1110

Feng Y, Cui R, Wang S, He M, Hua Y, Shi L, Ye X, Xu F (2020) Transcription factor BnaA9.WRKY47 contributes to the adaptation of Brassica napus to low boron stress by up-regulating the boric acid channel gene BnaA3.NIP5; 1. Plant Biotechnol J 18:1241-1254

Ferrándiz C, Liljegren SJ, Yanofsky MF (2000) Negative regulation of the SHATTERPROOF genes by FRUITFULL during Arabidopsis fruit development. Science 289:436-438

Fitt BDL, Hu BC, Li ZQ, Liu SY, Lange RM, Kharbanda PD, Butterworth MH, White RP (2008) Strategies to prevent spread of Leptosphaeria maculans (phoma stem canker) onto oilseed rape crops in China; costs and benefits. Plant Pathol 57:652-664

Gardiner DM, Howlett BJ (2004) Negative selection using thymidine kinase increases the efficiency of recovery of transformants with targeted genes in the filamentous fungus Leptosphaeria maculans. Curr Genet 45:249-255

Gaudelli NM, Komor AC, Rees HA, Packer MS, Badran AH, Bryson DI, Liu DR (2017) Programmable base editing of A.T to G.C in genomic DNA without DNA cleavage. Nature 551:464-471
Guo Y, Hans H, Christian J, Molina C (2014) Mutations in single FT-and TFL1-paralogs of rapeseed (Brassica napus L.) and their impact on flowering time and yield components. Front. Plant Sci 5:282

Hu X, Sullivan-Gilbert M, Gupta M, Thompson SA (2006) Mapping of the loci controlling oleic and linolenic acid contents and development of fad 2 and fad 3 allele-specific markers in canola (Brassica napus L.). Theor Appl Genet 113:497-507

Hu YR, Dong QY, Yu DQ (2012) Arabidopsis WRKY46 coordinates with WRKY70 and WRKY53 in basal resistance against pathogen Pseudomonas syringae. Plant Sci 185-186:288-297

Hu Z, Yang H, Zhang L, Wang X, Liu G, Wang H, Hua W (2015) A large replum-valve joint area is associated with increased resistance to pod shattering in rapeseed. Int J Plant Res 128:813-819

Huang H, Cui T, Zhang L, Yang Q, Yang Y, Xie K, Fan C, Zhou Y (2020) Modifications of fatty acid profile through targeted mutation at BnaFAD2 gene with CRISPR/Cas9-mediated gene editing in Brassica napus. Theor Appl Genet 133:2401-2411

Idnurm A, Urquhart AS, Vummadi DR, Chang S, Van de Wouw AP, López-Ruiz FJ (2017) Spontaneous and CRISPR/Cas9-induced mutation of the osmosensor histidine kinase of the canola pathogen Leptosphaeria maculans. Fung Biol Biotechnol 4:12

Iwabuchi M, Koizuka N, Fujimoto H, Sakai T, Imamura J (1999) Identification and expression of the kosena radish (Raphanus sativus cv. Kosena) homologue of the ogura radish CMS-associated gene, orf138. Plant Mol Biol 39:183-188

Jiang L, Li D, Jin L, Ruan Y, Shen WH, Liu C (2018) Histone lysine methyltransferases BnaSDG8.A and BnaSDG8.C are involved in the floral transition in Brassica napus. Plant J 95:672-685

Jaradat MR, Ruegger M, Bowling A, Butler H, Cutler AJ (2014) A comprehensive transcriptome analysis of silique development and dehiscence in Arabidopsis and Brassica integrating genotypic, interspecies and developmental comparisons. GM Crops Food 5:302320

Jiquel A, Gervais J, Geistodt-Kiener A, Delourme R, Gay EJ, Ollivier B, Fudal I, Faure S, Balesdent MH, Rouxel T (2021) A gene-for-gene interaction involving a 'late' effector contributes to quantitative resistance to the stem canker disease in Brassica napus. New Phytol DOI. https://doi.org/10.1111/nph.17292

Jofuku KD, Den Boer BG, Van Montagu M, Okamuro JK (1994) Control of Arabidopsis flower and seed development by the homeotic gene APETALA2. Plant Cell 6:1211-1225

Jørgensen T, Hauser TP, Jørgensen RB (2007) Adventitious presence of other varieties in oilseed rape (Brassica napus) from seed banks and certified seed. Seed Sci Res 17:115-125

Journot-Catalino N, Somssich IE, Roby D, Kroj T (2006) The transcription factors WRKY11 and WRKY17 act as negative regulators of basal resistance in Arabidopsis thaliana. Plant Cell 18:3289-3302

Jung JH, Ju Y, Seo PJ, Lee JH, Park CM (2012) The SOC1-SPL module integrates photoperiod and gibberellic acid signals to control flowering time in Arabidopsis. Plant J 69:577-588

Kadkol GP, Beliharz VC, Halloran GM, MacMillan RH (1986) Anatomical basis of shatter-resistance in the oilseed Brassica. Aust J Bot 34:595-601

Kang BC, Yun JY, Kim ST, Shin Y, Ryu J, Choi M, Woo JW, Kim JS (2018) Precision genome engineering through adenine base editing in plants. Nat Plants 4:427-431

Kardailsky I, Shukla VK, Ahn JH, Dagenais N, Christensen SK, Nguyen JT, Chory J, Harrison MJ, Weigel D (1999) Activation tagging of the floral inducer FT. Science 286:1962-1965

Karunarathna NL, Wang H, Harloff HJ, Jiang L, Jung C (2020) Elevating seed oil content in a polyploid crop by induced mutations in SEED FATTY ACID REDUCER genes. Plant Biotechnol J 18:2251-2266 
Kazama T, Okuno M, Watari Y, Yanase S, Koizuka C, Tsuruta Y, Sugaya H, Toyoda A, Itoh T, Tsutsumi N, Toriyama K (2019) Curing cytoplasmic male sterility via TALEN-mediated mitochondrial genome editing. Nat Plants 5:722-730

Kim W, Park TI, Yoo SJ, Jun AR, Ahn JH (2013) Generation and analysis of a complete mutant set for the Arabidopsis FT/TFL1 family shows specific effects on thermo-sensitive flowering regulation. J Exp Bot 64:1715-1729

Kochevenko A, Willmitzer L (2003) Chimeric RNA/DNA oligonucleotide-based site-specific modification of the tobacco acetolactate synthase gene. Plant Physiol 132:174-184

Kohno-Murase J, Murase M, Ichikawa H, Imamura J (1994) Effects of an antisense napin gene on seed storage compounds in transgenic Brassica napus seeds. Plant Mol Biol 26:1115-1124

Komor AC, Kim YB, Packer MS, Zuris JA, Liu DR (2016) Programmable editing of a target base in genomic DNA without double stranded DNA cleavage. Nature 533:420-424

Koornneef M, Van der Veen JH (1980) Induction and analysis of gibberellin sensitive mutants in Arabidopsis thaliana (L.) Heynh. Theor Appl Genet 58:257-263

Lai CP, Huang LM, Chen LFO, Chan MT, Shaw JF (2017) Genomewide analysis of GDSL-type esterases/lipases in Arabidopsis. Plant Mol Biol 95:181-197

Lee K, In Sohn S, Jung JH, Kim SH, Roh KH, Kim J, Suh MC, Kim HU (2013) Functional analysis and tissue-differential expression of four FAD2 genes in amphidiploid Brassica napus derived from Brassica rapa and Brassica oleracea. Gene 531:253-262

Li A, Jiang J, Zhang Y, Snowdon RJ, Liang G, Wang Y (2012a) Molecular and cytological characterization of introgression lines in yellow seed derived from somatic hybrids between Brassica napus and Sinapis alba. Mol Breed 29:209-219

Li C, Hao M, Wang W, Wang H, Chen F, Chu W, Zhang B, Mei D, Cheng H, Hu Q (2018a) An efficient CRISPR/Cas9 platform for rapidly generating simultaneous mutagenesis of multiple gene homoeologs in allotetraploid oilseed rape. Front Plant Sci 9:442

Li J, Zhang Y, Zhang Y, Yu P-L, Pan H, Rollins JA (2018b) Introduction of large sequence inserts by CRISPR-Cas9 to create pathogenicity mutants in the multinucleate filamentous pathogen Sclerotinia sclerotiorum. mBio 9:e0567-18. https://doi.org/10.1128/mBio. 00567-18, 9.

Li X, Chen L, Hong M, Zhang Y, Zu F, Wen J, Yi B, Ma C, Shen J, Tu J, $\mathrm{Fu} \mathrm{T} \mathrm{(2012b)} \mathrm{A} \mathrm{large} \mathrm{insertion} \mathrm{in} \mathrm{bHLH} \mathrm{transcription} \mathrm{factor} \mathrm{BrTT8}$ resulting in yellow seed coat in Brassica rapa. PLoS One 7:e44145

Liang X, Liberti D, Li M, Kim YT, Hutchens A, Wilson R, Rollins JA (2015) Oxaloacetate acetylhydrolase gene mutants of Sclerotinia sclerotiorum do not accumulate oxalic acid, but do produce limited lesions on host plants. Mol Plant Pathol 16:559-571

Liljegren SJ, Ditta GS, Eshed Y, Savidge B, Bowman JL, Yanofsky MF (2000) SHATTERPROOF MADS-box genes control seed dispersal in Arabidopsis. Nature 404:766-770

Liljegren SJ, Roeder AHK, Kempin SA, Gremski K, Ostergaard L, Guimil S, Reyes DK, Yanofsky MF (2004) Control of fruit patterning in Arabidopsis by INDEHISCENT. Cell 116:843-853

Lin CS, Hsu CT, Yang LH, Lee LY, Fu JY, Cheng QW, Wu FH, Hsiao HC, Zhang Y, Zhang R, Chang WJ (2018) Application of protoplast technology to CRISPR/Cas9 mutagenesis: from single-cell mutation detection to mutant plant regeneration. Plant Biotechnol J 16:12951310

Lin Q, Zong Y, Xue C, Wang S, Jin S, Zhu Z, Wang Y, Anzalone AV, Raguram A, Doman JL, Liu DR (2020) Prime genome editing in rice and wheat. Nat Biotechnol 38:582-585

Liu F, Xiong X, Wu L, Fu D, Hayward A, Zeng X, Cao Y, Wu Y, Li Y, Wu G (2014) BraLTP1, a lipid transfer protein gene involved in epicuticular wax deposition, cell proliferation and flower development in Brassica napus. PLoS One 9:e110272

Liu J, Wang J, Wang H, Wang W, Zhou R, Mei D, Cheng H, Yang J, Raman H, Hu Q (2016) Multigenic control of pod shattering resistance in Chinese rapeseed germplasm revealed by genome-wide association and linkage analyses. Front Plant Sci 7:1058

Lu W, Liu J, Xin Q, Wan L, Hong D, Yang G (2012) A triallelic genetic male sterility locus in Brassica napus: an integrative strategy for its physical mapping and possible local chromosome evolution around it. Ann Bot 111:305-315

Mao Y, Botella JR, Liu Y, Zhu JK (2019) Gene editing in plants: progress and challenges. Natl Sci Rev 6:421-437

Marschner P (2012) Marschner's mineral nutrition of higher plants, 3rd edn. Academic Press, London

Mishra R, Joshi RK, Zhao K (2020) Base editing in crops: current advances, limitations and future implications. Plant Biotechnol J 18: $20-31$

Morgan CL, Ladbrooke ZL, Bruce DM, Child R (2000) Breeding oilseed rape for pod shattering resistance. J Agric Sci 135:347-359

Murray GM, Brennan JP (2012) The current and potential costs from diseases of oilseed crops in Australia. Grains Research and Development Corporation https://grdc.com.au/_data/assets/pdf_ file/0021/82641/grdcreportdiseasecostoilseedspdf.pdf.pdf

Nagaharu U (1935) Genome analysis in Brassica with special reference to the experimental formation of B. napus and peculiar mode of fertilization. J Jap Bot 7:389-452

Nasrallah JB (1997) Evolution of the Brassica self-incompatibility locus: a look into S-locus gene polymorphisms. Proc Natl Acad Sci USA 94:9516-9519

Okuzaki A, Ogawa T, Koizuka C, Kaneko K, Inaba M, Imamura J, Koizuka N (2018) CRISPR/Cas9-mediated genome editing of the fatty acid desaturase 2 gene in Brassica napus. Plant Physiol Biochem 131:63-69

Okuzaki A, Toriyama K (2004) Chimeric RNA/DNA oligonucleotidedirected gene targeting in rice. Plant Cell Rep 22:509-512

Padmaja LK, Agarwal P, Gupta V, Mukhopadhyay A, Sodhi YS, Pental D, Pradhan AK (2014) Natural mutations in two homoeologous TT8 genes control yellow seed coat trait in allotetraploid Brassica juncea (AABB). Theor Appl Genet 127:339-347

Parlange F, Daverdin G, Fudal I, Kuhn ML, Balesdent MH, Blaise F, Grezes-Besset B, Rouxel T (2009) Leptosphaeria maculans avirulence gene AvrLm4-7 confers a dual recognition specificity by the Rlm 4 and $R \operatorname{lm} 7$ resistance genes of oilseed rape, and circumvents $\mathrm{R} 1 \mathrm{~m} 4$-mediated recognition through a single amino acid change. Mol Microbiol 71:851-863

Parkin IA, Sharpe AG, Keith DJ, Lydiate DJ (1995) Identification of the $\mathrm{A}$ and $\mathrm{C}$ genomes of amphidiploid Brassica napus (oilseed rape). Genome 38:1122-1131

Pien S, Fleury D, Mylne JS, Crevillen P, Inzé D, Avramova Z, Dean C, Grossniklaus U (2008) Arabidopsis TRITHORAX1 dynamically regulates FLOWERING LOCUS C activation via histone 3 lysine 4 trimethylation. Plant Cell 20:580-588

Pröbsting M, Schenke D, Hossain R, Häder C, Thurau T, Wighardt L, Schuster A, Zhou Z, Ye W, Rietz S, Leckband G (2020) Loss of function of CRT1a (calreticulin) reduces plant susceptibility to Verticillium longisporum in both Arabidopsis thaliana and oilseed rape (Brassica napus). Plant Biotechnol J 18:2328-2344

Purdy L (1979) Sclerotinia sclerotiorum: history, diseases and symptomatology, host range, geographic distribution, and impact. Phytopathology 69:875-880

Raboy V (2009) Approaches and challenges to engineering seed phytate and total phosphorus. Plant Sci 177:281-296 
Raman H, Raman R, Kilian A, Detering F, Carling J, Coombes N, Diffey S, Kadkol G, Edwards D, McCully M, Ruperao P, Parkin IAP, Batley J, Luckett DJ, Wratten N (2014) Genome-wide delineation of natural variation for pod shatter resistance in Brassica napus. PLoS ONE 9:e101673

Rajani S, Sundaresan V (2001) The Arabidopsis myc/bHLH gene ALCATRAZ enables cell separation in fruit dehiscence. Curr Biol 11:1914-1922

Razzaq A, Saleem F, Kanwal M, Mustafa G, Yousaf S, Arshad HMI, Hameed MK, Khan MS, Joyia FA (2019) Modern Trends in plant genome editing: an inclusive review of the CRISPR/Cas9 toolbox. Int J Mol Sci 20:40-45

Rodríguez-Leal D, Lemmon ZH, Man J, Bartlett ME, Lippman ZB (2017) Engineering quantitative trait variation for crop improvement by genome editing. Cell 171:470-480

Sakai T, Imamura J (1992) Alteration of mitochondrial genomes containing atpA genes in the sexual progeny of cybrids between Raphanus sativus cms line and Brassica napus cv. Westar. Theor Appl Genet 84:923-929

Sashidhar N, Harloff HJ, Potgieter L, Jung C (2020) Gene editing of three BnITPK genes in tetraploid oilseed rape leads to significant reduction of phytic acid in seeds. Plant Biotechnol J 18:2241-2250

Schopfer CR, Nasrallah ME, Nasrallah JB (1999) The male determinant of self-incompatibility in Brassica. Science 286:1697-1700

Schruff MC, Spielman M, Tiwari S, Adams S, Fenby N, Scott RJ (2006) The AUXIN RESPONSE FACTOR 2 gene of Arabidopsis links auxin signaling, cell division, and the size of seeds and other organs. Development 133:251-261

Sharafi Y, Majidi MM, Goli SA, Rashidi F (2015) Oil content and fatty acids composition in Brassica species. Int J Food Prop 18:21452154

Shi S, Gao Q, Zeng J, Liu X, Pu Q, Liu G, Zhang H, Yang X, Zhu L (2016) N-terminal domains of ARC1 are essential for interaction with the N-terminal region of Exo70A1 in transducing selfincompatibility of Brassica oleracea. Acta Biochim Biophys Sin 48:777-787

Silverstone AL, Ciampaglio CN, Sun TP (1998) The Arabidopsis RGA gene encodes a transcriptional regulator repressing the gibberellin signal transduction pathway. Plant Cell 10:155-169

Silverstone AL, Mak PYA, Martinez EC, Sun TP (1997) The new RGA locus encodes a negative regulator of gibberellin response in Arabidopsis thaliana. Genetics 146:1087-1099

Snowdon RJ, Friedrich T, Friedt W, Köhler W (2002) Identifying the chromosomes of the A-and C-genome diploid Brassica species $B$. rapa (syn. campestris) and B. oleracea in their amphidiploid B. napus. Theor Appl Genet 104:533-538

Sriboon S, Li H, Guo C, Senkhamwong T, Dai C, Liu K (2020) Knockout of TERMINAL FLOWER 1 genes altered flowering time and plant architecture in Brassica napus. BMC Genet 21:1-3

Sun Q, Lin L, Liu D, Wu D, Fang Y, Wu J, Wang Y (2018) CRISPR/ Cas9-mediated multiplex genome editing of the BnWRKY11 and BnWRKY70 Genes in Brassica napus L. Int J Mol Sci 19:2716

Stanic M, Hickerson NM, Arunraj R, Samuel MA (2020) Gene-editing of the strigolactone receptor BnD14 confers promising shoot architectural changes in Brassica napus (canola). Plant Biotechnol J:1-3

Suzuki G, Kai N, Hirose T, Fukui K, Nishio T, Takayama S, Isogai A, Watanabe M, Hinata K (1999) Genomic organization of the S locus: identification and characterization of genes in SLG/SRK region of S9 haplotype of Brassica campestris (syn. rapa). Genetics 153:391400

Tang T, Yu X, Yang H, Gao Q, Ji H, Wang Y, Yan G, Peng Y, Luo H, Liu K, Li X (2018) Development and validation of an effective CRISPR/Cas9 vector for efficiently isolating positive transformants and transgene-free mutants in a wide range of plant species. Front Plant Sci 9:1533

Van Eck J (2020) Applying gene editing to tailor precise genetic modifications in plants. J Biol Chem 295:13267-13276

Weigel D, Meyerowitz EM (1994) The ABCs of floral homeotic genes. Cell 78:203-209

Well R, Trick M, Soumpourou E, Clissold L, Morgan C, Bancroft I (2014) The control of seed oil polyunsaturate content in the polyploid crop species Brassica napus. Mol Breed 33:349-362

Wu J, Chen C, Xian G, Liu D, Lin L, Yin S, Sun Q, Fang Y, Zhang H, Wang Y (2020a) Engineering herbicide-resistant oilseed rape by CRISPR/Cas9-mediated cytosine base-editing. Plant Biotechnol J 18:1857-1859

Wu J, Yan G, Duan Z, Wang Z, Kang C, Guo L, Liu K, Tu J, Shen J, Yi $\mathrm{B}, \mathrm{Fu} \mathrm{T}$ (2020b) Roles of the Brassica napus DELLA protein BnaA6.RGA, in modulating drought tolerance by interacting with the ABA signaling component BnaA10.ABF2. Front. Plant Sci 11: 577

Wu J, Zhao Q, Yang Q, Liu H, Li Q, Yi X, Cheng Y, Guo L, Fan C, Zhou Y (2016) Comparative transcriptomic analysis uncovers the complex genetic network for resistance to Sclerotinia sclerotiorum in Brassica napus. Sci Rep 6:1-6

Xie T, Chen X, Guo T, Rong H, Chen Z, Sun Q, Batley J, Jiang J, Wang Y (2020) Targeted Knockout of BnTT2 homologues for yellowseeded Brassica napus with reduced flavonoids and improved fatty acid composition. J Agric Food Chem 68:5676-5690

Xie X, Yoneyama K, Yoneyama K (2010) The strigolactone story. Annu Rev Phytopathol 48:93-117

Xin Q, Shen Y, Li X, Lu W, Wang X, Han X, Dong F, Wan L, Yang G, Hong D, Cheng Z (2016) MS5 mediates early meiotic progression and its natural variants may have applications for hybrid production in Brassica napus. Plant Cell 28:1263-1278

Xin Q, Wang X, Gao Y, Xu D, Xie Z, Dong F, Wan L, Yang L, Yang G, Hong D (2020) Molecular mechanisms underpinning the multiallelic inheritance of MS5 in Brassica napus. Plant J 103: $1723-1734$

Xu FS, Wang YH, Ying WH, Meng JL (2002) Inheritance of boron nutrition efficiency in Brassica napus. J Plant Nutr 25:901-912

Xu L, Zhao Z, Dong A, Soubigou-Taconnat L, Renou JP, Steinmetz A, Shen WH (2008) Di-and tri-but not monomethylation on histone H3 lysine 36 marks active transcription of genes involved in flowering time regulation and other processes in Arabidopsis thaliana. Mol Cell Biol 28:1348-1360

Yamaguchi A, Wu MF, Yang L, Wu G, Poethig RS, Wagner D (2009) The microRNA-regulated SBP-Box transcription factor SPL3 is a direct upstream activator of LEAFY, FRUITFULL, and APETALA1. Dev Cell 17:268-278

Yang H, Wu JJ, Tang T, Liu KD, Dai C (2017) CRISPR/Cas9-mediated genome editing efficiently creates specific mutations at multiple loci using one sgRNA in Brassica napus. Sci Rep 7:1-3

Yang Q, Fan C, Gua Z, Qin J, Wu J, Li Q, Fu T, Zhou Y (2012) Identification of FAD2 and FAD3 genes in Brassica napus genome and development of allele-specific markers for high oleic and low linoleic acid contents. Theor Appl Genet 125:715-729

Yang Y, Zhu K, Li H, Han S, Meng Q, Khan SU, Fan C, Xie K, Zhou Y (2018) Precise editing of CLAVATA genes in Brassica napus L. regulates multilocular silique development. Plant Biotechnol J 16: 1322-1335

Zaman QU, Chu W, Hao M, Shi Y, Sun M, Sang SF, Mei D, Cheng H, Liu J, Li C, Hu Q (2019) CRISPR/Cas9-mediated multiplex genome editing of JAGGED gene in Brassica napus L. Biomolecules 9:725

Zhai Y, Cai S, Hu L, Yang Y, Amoo O, Fan C, Zhou Y (2019) CRISPR/ Cas9-mediated genome editing reveals differences in the 
contribution of INDEHISCENT homologues to pod shatter resistance in Brassica napus L. Theor Appl Genet 132:2111-2123

Zhai Y, Yu K, Cai S, Hu L, Amoo O, Xu L, Yang Y, Ma B, Jiao Y, Zhang C, Khan MH (2020) Targeted mutagenesis of BnTT8 homologs controls yellow seed coat development for effective oil production in Brassica napus L. Plant Biotechnol J 18:1153-1168

Zhang B, Liu C, Wang Y, Yao X, Wang F, Wu J, King GJ, Liu K (2015) Disruption of a CAROTENOID CLEAVAGE DIOXYGENASE 4 gene converts flower colour from white to yellow in Brassica species. New Phytol 206:1513-1526

Zhang K, Nie L, Cheng Q, Yin Y, Chen K, Qi F, Zou D, Liu H, Zhao W, Wang B, Li M (2019) Effective editing for lysophosphatidic acid acyltransferase $2 / 5$ in allotetraploid rapeseed (Brassica napus L.) using CRISPR-Cas9 system. Biotechnol Biofuels 12:1-8

Zhang X, Peng G, Kutcher HR, Balesdent MH, Delourme R, Fernando WGD (2016) Breakdown of Rlm3 resistance in the Brassica napus Leptosphaeria maculans pathosystem in western Canada. Eur J Plant Pathol 145:659-674

Zhang Y, Huang S, Wang X, Liu J, Guo X, Mu J, Tian J, Wang X (2018) Defective APETALA2 genes lead to sepal modification in Brassica crops. Front Plant Sci 20:367

Zhao B, Li H, Li J, Wang B, Dai C, Wang J, Liu K (2017) Brassica napus DS-3, encoding a DELLA protein, negatively regulates stem elongation through gibberellin signaling pathway. Theor Appl Genet 130:727-741

Zheng M, Zhang L, Tang M, Liu J, Liu H, Yang H, Fan S, Terzaghi W, Wang H, Hua W (2020) Knockout of two BnaMAX1 homologs by CRISPR/Cas9-targeted mutagenesis improves plant architecture and increases yield in rapeseed (Brassica napus L.). Plant Biotechnol J 18:644-654

Zhou YM, Wang H, Gilmer S, Whitwill S, Keller W, Fowke LC (2002) Control of petal and pollen development by the plant cyclin dependent kinase inhibitor ICK1 in transgenic Brassica plants. Planta 215: 248-257

Zhu T, Mettenburg K, Peterson DJ, Tagliani L, Baszczynski CL (2000) Engineering herbicide-resistant maize using chimeric RNA/DNA oligonucleotides. Nat Biotechnol 18:555-558

Zhu T, Peterson DJ, Tagliani L, Clair GS, Baszczynski CL, Bowen B (1999) Targeted manipulation of maize genes in vivo using chimeric RNA/DNA oligonucleotides. Proc Natl Acad Sci USA 96:87688773

Zou Z, Liu F, Selin C, Fernando WG (2020) Generation and characterization of a virulent Leptosphaeria maculans isolate carrying a mutated AvrLm7 gene using the CRISPR/Cas9 system. Front Microbiol 11:1969 